\title{
Explaining clusterings of process instances
}

\author{
Pieter De Koninck • Jochen De Weerdt • \\ Seppe K. L. M. vanden Broucke
}

Received: date / Accepted: date

\begin{abstract}
This paper presents a technique that aims to increase human understanding of trace clustering solutions. The clustering techniques under scrutiny stem from the process mining domain, where the clustering of process instances is deemed a useful technique to analyse process data with a large variety of behaviour. Until now, the most often used method to inspect clustering solutions in this domain is visual inspection of the clustering results. This paper proposes a more thorough approach based on the post hoc application of supervised learning with support vector machines on cluster results. Our approach learns concise rules to describe why a specific instance is included in a certain cluster based on specific control-flow based feature variables. An extensive experimental evaluation is presented showing that our technique outperforms alternatives. Likewise, we are able to identify features that lead to shorter and more accurate explanations.
\end{abstract}

Keywords process discovery · trace clustering · human understanding . instance-level explanations $\cdot$ support vector machines

\section{Introduction}

Within the process mining domain (van der Aalst, 2016), algorithms for automatically discovering process models from event logs often don't cope well with complex input, i.e. those containing a large variety of behaviour. Partitioning event logs into multiple groups is a convenient approach for addressing the challenge of discovering accurate and understandable process models from logs presenting a large amount of distinct process behaviour. Partitioning algorithms in the process mining domain are referred to as trace clustering techniques. In this paper, we aim at improving the application potential of trace clustering by proposing a technique to provide better human understanding of trace clustering results. Observe that the importance of human understanding is neither restricted to clustering of traces nor to unsupervised learning (Ribeiro et al, 2016).

The process mining domain is to be situated at the intersection of the fields of Business Process Management (BPM) (Dumas et al, 2013) and Knowledge Discovery in Databases (KDD) (Fayyad et al, 1996). It is of value as it provides a new, actionable approach in the context of data-driven business process analysis and

Pieter De Koninck $(\varangle)$

KU Leuven - University of Leuven, Research Centre for Management Informatics, Faculty of Economics and Business, Naamsestraat 69, B-3000 Leuven, Belgium

E-mail: pieter.dekoninck@kuleuven.be

Jochen De Weerdt

KU Leuven - University of Leuven, Research Centre for Management Informatics, Faculty of Economics and Business, Naamsestraat 69, B-3000 Leuven, Belgium

E-mail: jochen.deweerdt@kuleuven.be

Seppe K. L. M. vanden Broucke

KU Leuven - University of Leuven, Research Centre for Management Informatics, Faculty of Economics and Business, Naamsestraat 69, B-3000 Leuven, Belgium

E-mail: seppe.vandenbroucke@kuleuven.be 
improvement. Typically, organisational units that are concerned with BPM make use of models for explaining, designing, and reengineering business processes. Such models are often represented visually, with modeling notations such as the Business Process Modeling Notation (BPMN), Event-driven Process Chains (EPC), or Petri nets. In a nutshell, the essence of these notations is the visual representation of control-flow constraints on activities undertaken within a particular business process. An example Petri net of a simple loan application process is shown in Figure 1.

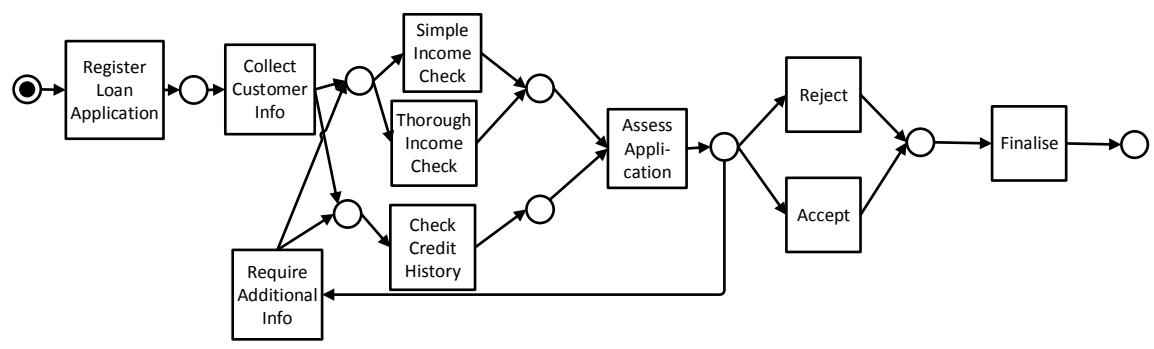

Fig. 1 An example Petri net of a loan application process

While BPM has been around for a few decades, the attention within the domain has recently shifted towards data-driven analysis of business processes, grouped under the umbrella term Process Mining (van der Aalst, 2016). Process mining techniques become more and more valuable as the automatic registration of trails of the execution of everyday activities undertaken within organisations is increasingly incorporated in modern information systems. As such, these trails, commonly termed as an event log in the process mining domain, contain valuable information concerning the execution of business processes. Therefore, the goal of process mining techniques is to derive knowledge from event logs, for instance by discovering the actual process, checking compliance, or deriving guidelines for improvements. The key enabler of this goal is process discovery entailing the automated extraction of a process model from an event log representing the behaviour in the log as closely as possible. A multitude of process discovery algorithms have been proposed, however, it is not the goal of this work to present an overview of these techniques. For the interested reader, we refer to (Cook and Wolf, 1998, van der Aalst et al, 2004, Weijters et al, 2006, de Medeiros et al, 2007, Goedertier et al, 2009, Sole and Carmona, 2011).

As explained above, various trace clustering techniques (Greco et al, 2006, Song et al, 2008, Ferreira et al, 2007, Bose and van der Aalst, 2009, 2010; Folino et al, 2011: De Weerdt et al, 2013, Ekanayake et al, 2013; Delias et al, 2015) have been devised to deal with complex event logs containing a too wide variety of distinct process instances so that the application of existing process discovery techniques would usually result in one inaccurate and overly complex model. However, the uptake of trace clustering in practical settings is curtailed by the low level of human understanding of trace clustering results.

Concretely, there exist two major problems regarding trace clustering solutions. First, it is non-trivial to find out what the driving elements are that determine a clustering technique to split up the event $\log$ in a particular way. This is because most trace clustering techniques operate at a higher level of abstraction which makes that, for instance, the concept of distance, an often used metric in learning algorithms in general (Wang and Sun, 2014), between traces is not very insightful as a means to describe a clustering solution. Secondly, end users would like to be able to understand the differentiating characteristics between multiple clusters of process instances, preferably from a domain perspective, i.e. relying on control-flow characteristics that are present in the context of the process at hand.

Therefore, SECPI (Search for Explanations of Clusters of Process Instances) was presented in (De Weerdt and Vanden Broucke, 2014), an algorithm that is capable of finding a minimal set of control-flow characteristics for a process instance, such that if these characteristics were not present, the process instance 
would not remain within its current cluster. Furthermore, its implementation allows to visualise explanations in the respective process models so that users can easily observe what characteristics make that a process instance belongs to a certain cluster. This paper contributes by extending (De Weerdt and Vanden Broucke, 2014) as follows: first, it incorporates a thorough overview of potential alternatives to SECPI (Section 2.2). It is argued that instance-level explanations can overcome drawbacks of potential alternative explanation techniques, such as for example the visual analysis of the underlying process models. Secondly, this paper discusses and evaluates different alternative feature sets that can be used as input for an explanatory technique (Section 3.1). As a third contribution, the capability of our technique is extensively evaluated using real-life datasets (Section 4). This experimental evaluation consists of two main parts: in a first part, our technique is compared to white-box classification methods using varying feature sets. Our technique is shown to strike a better balance between accurate representation of the clusters, and comprehensibility. In a second part, it is shown how our implementation can support comparisons between clustering solutions. Observe that the extensive experimental setup also led to a finetuning of the original algorithm, as discussed in Section 3 .

Accordingly, this paper is organised as follows. Section 2 provides an overview of trace clustering techniques and further details the problem at hand. Furthermore, a number of potential alternative cluster explanation techniques are discussed as well as useful notation, and other related work. Afterwards, the SECPIalgorithm and its input are described in Section 3. Finally, Section 4 presents an extensive experimental evaluation before conclusions are formulated in Section 5.

\section{Preliminaries}

\subsection{Trace Clustering}

Trace clustering is an interesting approach to deal with the problem that many event logs contain an extensive amount of distinct behaviour (i.e. process variants), because it allows the user to split up a log so that multiple distinct models can be learnt to describe the underlying business process. Table 1 shows that several currently available trace clustering techniques translate the learning problem into a propositional setting by converting an event log into an attribute-value data set, to which well-known clustering techniques such as k-means or hierarchical clustering can be applied. The pioneering study by Greco et al (2006) proposes a projection of the traces onto a set of suitable features, in this case a set of frequent k-grams of activity sequences. The projection of an event log onto a feature set was further explored by Song et al (2008). Their trace clustering implementation allows for a multitude of profiles to determine the vector associated with each process instance. As such, they define activity, transition, performance, case attribute profiles, etc. Furthermore, whereas Greco et al (2006) relied on k-means, Song et al (2008) provide a full range of distance metrics and clustering algorithms. Recently, the use of dimensionality reduction techniques was investigated in the context of profile-based trace clustering (Song et al, 2013). Along the same line of reasoning, Bose and van der Aalst (2009, 2010) propose two additional trace clustering techniques. In (Bose and van der Aalst, 2010), the authors refine the technique of Greco et al (2006) by making use of so-called conserved patterns (e.g. Maximal, Super Maximal, and Near Super Maximal Repeats) as projection features. The implementation applies hierarchical clustering instead of k-means. In (Bose and van der Aalst, 2009), Bose and van der Aalst turn away from the use of an attribute-value feature space, but instead regard log traces as strings over the alphabet of task labels and as such calculate the distance between traces directly by exploiting the idea of a string edit distance. However, in contrast to contemporary approaches such as the Levenshtein distance (Levenshtein, 1966), specific substitution, insertion and deletion costs are derived so as to take into account characteristic features of an event log, such as concurrency of tasks. A similar, alignment-based technique is proposed in (Evermann et al, 2016). Furthermore, Delias et al (2015) have developed a more robust spectral learning approach, where 
the similarity metric is adjusted in the face of significant deviating behaviour. Finally, Appice and Malerba (2015) deploy a co-training strategy based on multiple views: separate clusterings are created using instance similarity based on the activities, resources, sequences and timing aspects of the process instances, and then combined to create a single multiple-view clustering.

A second class of trace clustering techniques can be best described as modeldriven. Inspired by the work of Cadez et al (2003) in the area of web usage mining, Ferreira et al (2007) propose to cluster sequences by learning a mixture of firstorder Markov models using the Expectation-Maximization (EM) algorithm. This technique has been applied in (Veiga and Ferreira, 2010) to server logs. Also in (Folino et al, 2011), Markov chain clustering is used. Finally, De Weerdt et al (2013) propose the ActiTraC-algorithm, which strives to optimise the combined fitness of the underlying process models.

Table 1 Available trace clustering techniques and their characteristics

\begin{tabular}{|c|c|c|c|}
\hline Author & $\begin{array}{l}\text { Data } \\
\text { Representation }\end{array}$ & $\begin{array}{l}\text { Clustering } \\
\text { Technique }\end{array}$ & Clustering Bias \\
\hline Greco et al (2006) & propositional & k-means & $\begin{array}{l}\text { instance similarity: } \\
\text { alphabet k-grams }\end{array}$ \\
\hline Song et al 2008) & propositional & various & $\begin{array}{l}\text { instance similarity: } \\
\text { profiles }\end{array}$ \\
\hline Ferreira et al (2007) & event log & $\begin{array}{l}\text { first order Markov } \\
\text { mixture model by } \\
\text { EM }\end{array}$ & maximum likelihood \\
\hline $\begin{array}{l}\text { Bose and van der } \\
\text { Aalst }(2009)\end{array}$ & event $\log$ & $\begin{array}{l}\text { hierarchical } \\
\text { clustering }\end{array}$ & $\begin{array}{l}\text { instance similarity: } \\
\text { string edit distance }\end{array}$ \\
\hline $\begin{array}{l}\text { Bose and van der } \\
\text { Aalst }(2010)\end{array}$ & propositional & $\begin{array}{l}\text { hierarchical } \\
\text { clustering }\end{array}$ & $\begin{array}{l}\text { instance similarity: } \\
\text { conserved patterns }\end{array}$ \\
\hline Folino et al (2011) & event log & $\begin{array}{l}\text { enhanced Markov } \\
\text { cluster model }\end{array}$ & maximum likelihood \\
\hline De Weerdt et al & event $\log$ & $\begin{array}{l}\text { model-driven } \\
\text { clustering }\end{array}$ & $\begin{array}{l}\text { combined process } \\
\text { model fitness (ICS) }\end{array}$ \\
\hline $\begin{array}{l}\text { Ekanayake et al } \\
(2013)\end{array}$ & propositional & $\begin{array}{l}\text { complexity-aware } \\
\text { clustering }\end{array}$ & $\begin{array}{l}\text { instance similarity } \\
+ \text { repository } \\
\text { complexity }\end{array}$ \\
\hline Delias et al (2015) & event $\log$ & spectral & $\begin{array}{l}\text { robust instance } \\
\text { similarity }\end{array}$ \\
\hline $\begin{array}{l}\text { Appice and Malerba } \\
(2015)\end{array}$ & event $\log$ & co-training & $\begin{array}{l}\text { activity, sequence, } \\
\text { performance and } \\
\text { resource } \\
\text { perspectives }\end{array}$ \\
\hline$\frac{\text { Evermann et al }}{2016}$ & event $\log$ & k-means & $\begin{array}{l}\text { instance similarity: } \\
\text { SWG-alignment }\end{array}$ \\
\hline
\end{tabular}

\subsection{Potential Alternative Cluster Explanation Techniques}

Before outlining our instance-level cluster explanation approach in the next section, five alternative techniques, potentially useful for cluster explanation, are explored. These approaches can be described as global explanation methods because they seek to provide human understanding from a general viewpoint, i.e. aggregating over the entire set of process instances. This section points out opportunities and disadvantages of alternative explanation techniques, so that we can adequately argue for and position our novel approach.

The most obvious method for obtaining human understanding of a trace clustering result is a visual comparative analysis of the underlying discovered process models. Although this is an intuitive solution, different drawbacks exist. First of 
all, identifying dissimilarities between two process models often requires a high level of expertise, and is even further complicated by the fact that differences have to be identified for a set of process models. Secondly, the process models have to be discovered before they can be inspected. Therefore, the chosen discovery technique might influence the visualisation. Furthermore, the discovered models might still be too complex to interpret visually.

A second solution for cluster characterisation is the use of automated similarity analysis techniques for business process models. There exists an extensive body of literature on the behavioural equivalence of process models van Glabbeek and Goltz, 2001; Hidders et al, 2005). Common notions such as trace equivalence, bisimulation, and branching bisimulation can be used to compare process models (Kiepuszewski et al, 2003). However, most of these equivalence notions only provide the user with a strict true or false answer, as pointed out in van der Aalst et al, 2006), which makes them inapplicable to our context. Nevertheless, other researchers such as de Medeiros et al (2008) or Dijkman et al (2011) have proposed more nuanced similarity metrics for comparing business process models, which potentially better suit the problem at hand. The main downside with regards to human understanding of any of these metrics is that they do not provide details on the root causes of dissimilarity.

As a third potential alternative cluster explanation technique, Dijkman proposed a process similarity technique that is capable of returning the exact positions of differences between two process models (Dijkman, 2008) by means of typology of process model differences defined in (Dijkman, 2007). Compared to the previous alternative explanation technique, this technique has the advantage to be capable of identifying root causes of process model differences. Observe however that the technique is specifically designed to diagnose Event-Driven Process Chains (van der Aalst, 1999). Furthermore, the technique has exponential complexity and can only compare two models at a time.

A fourth potential cluster explanation technique is the construction of a white box classification model. In fact, this approach corresponds to a reverse engineering of conventional trace clustering techniques where process instances are projected onto feature vectors. One could apply rule learning or decision tree algorithms to a data set obtained by combining the feature vectors and the cluster identifier. As such, a classification model can be learnt that explains why process instances belong to a certain cluster. White box classification techniques such as RIPPER (Cohen, 1995) and C4.5 decision trees (Quinlan, 1993) have been shown to be useful in the process mining domain, e.g. in (Rozinat and van der Aalst, 2006). However, the usefulness of such classification models is determined by two important parameters. First of all, the model should present a high classification accuracy so as to be valid. Furthermore, the model should be comprehensible for end users. In Section 4, an extensive experiment is presented comparing white box classification techniques to our newly proposed algorithm.

The last potential alternative technique for providing human understanding to a trace clustering solution is the use of conformance checking (Rozinat and van der Aalst, 2008), for instance through model-log alignment algorithm (Adriansyah et al, 2011). In this particular case, one could rely on cross-cluster model-log alignment for identifying differences between clusters. By aligning an entire cluster log with the different process models underlying the other clusters in the clustering solution, it is feasible to visualise differences at a global level. Furthermore, this approach could also be performed on an instance-level basis. The main downside of this approach is that it requires discovered process models, something our approach avoids.

\subsection{Event logs as input data}

Event logs consist of events that pertain to process instances. A process instance is defined as a logical grouping of activities whose state changes are recorded as events. Furthermore, it is assumed that each event refers to an activity type, i.e. a step in a process instance, a case, i.e. a process instance identifier, and that events are totally ordered. Events are of a particular event type, which inherently relates 
to activity state changes as defined in enactment specifications of business process management systems or defined by the information system. Typical event types encountered in event logs are e.g. start, assign, skip, complete, etc. In reality however, many event logs only contain one event type per activity type, typically the complete-event is recorded, a situation which is also assumed in this work. As such, a process instance can be represented as a finite ordered subset of the activity alphabet of which the process consists. An example event log is shown in Table 2, A formal description of event logs can be found in the XES-standard (Günther and Verbeek, 2014).

Table 2 Example event $\log$ for a simple loan application system

\begin{tabular}{rllll}
\hline Case ID & Time & Activity Type & Event Type & Resource \\
\hline 1 & $30-12-2015$ 10:15:07 & Register Loan & Started & John \\
1 & $30-12-2015$ 10:18:24 & Register Loan & Completed & John \\
1 & $30-12-2015$ 10:18:45 & Collect Info & Started & John \\
1 & $30-12-2015$ 10:36:32 & Collect Info & Completed & John \\
1 & $31-12-2015$ 09:05:34 & Check credit & Started & Sara \\
1 & $31-12-2015$ 10:05:51 & Simple Income Check & Started & John \\
1 & $31-12-2015$ 11:00:09 & Simple Income Check & Completed & John \\
1 & $31-12-2015$ 11:20:44 & Check credit & Completed & Sara \\
1 & $31-12-2015$ 13:00:03 & Assess Application & Started & Sara \\
1 & $31-12-2015$ 13:10:49 & Assess Application & Completed & Sara \\
1 & $31-12-2015$ 14:20:28 & Reject & Completed & Sara \\
1 & $02-01-2016 ~ 09: 20: 13$ & Finalise & Completed & John \\
\hline 2 & $30-12-2015: 10: 37$ & Register Loan & Started & John \\
2 & $30-12-2015: 10: 40$ & Register Loan & Completed & John \\
2 & $\ldots$ & $\ldots$ & $\ldots$ & $\ldots$ \\
\hline
\end{tabular}

\subsection{Related work in other fields}

The challenge posed by understandability of clustering is not new, and alternative approaches for creating understandable clusterings or for extracting explanations have been described. Three categories are detailed in this section: first, approaches that aim to create understandable clusterings; second, approaches that aim to extract explanations from other types of models that are too complex to be directly interpreted; and thirdly, approaches that aim to delineate understandable features.

Conceptual clustering as described in (Michalski and Stepp, 1983, Fisher, 1987), amongst others, is a subfield of unsupervised learning in which cluster solutions are created with the following idea in mind: the quality of clusters does not only depend on the instances that are included in each cluster, but also on the concepts that can describe the elements in a cluster. Simple concepts should suffice to represent the content of a cluster. Related to the field of conceptual clustering is the fields of explanation-based learning (Mitchell et al, 1986). Here, the focus lies on constructing a concept from a set of training examples such that a concept definition can be formulated based on the essential common features of the examples. Generally speaking, such approaches result in a cluster-wide description of elements pertain in their cluster, whereas the aim in this paper is to provide individual explanations.

Secondly, our research is related to the domain that is concerned with the extraction of understandable explanations from models which are beyond human understanding, often in the form of rule extraction: from support vector machines (Martens et al, 2007), neural networks (Andrews et al, 1995), or any classifier (Ribeiro et al, 2016$)$.

In order to extract rules or generate explanations, one needs a feature space. In our approach, this is done in a fixed manner, to provide understandable and visualizable explanations. Nonetheless, one could deploy a method where these features are induced in a more natural way, using approaches from inductive logic programming to induce such features. Such approaches have been used in the process mining domain, mainly for the representation and discovery of process models (Lamma et al, 2008; Chesani et al, 2009). 


\section{Instance-Level Explanations with SECPI}

\subsection{Rationale}

The core idea is shown in Figure 2 Instead of providing a global explanation, concise rules are learnt for each individual instance, i.e. a list of control-flow characteristics (e.g. "sometimes directly follows" relations, see Table 3), from a black box support vector machine (SVM) model. This list of characteristics are the determining factors that make that a certain instance pertains to its current cluster, given the representational bias of the investigated features. Experiments presented in the next section show that the overall accuracy of our explanatory techniques using SVMs is high, and the resulting rules are indeed concise in terms of the number of characteristics.

\section{Trace clustering}

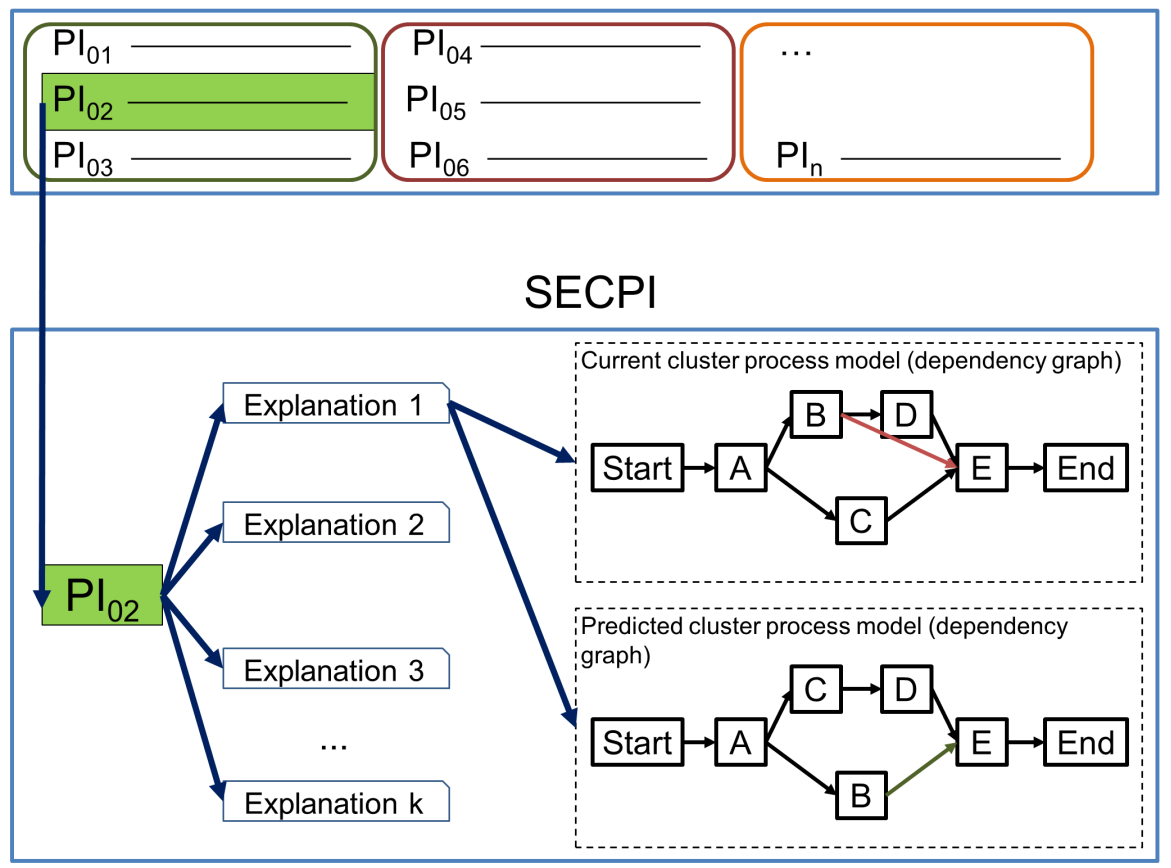

Fig. 2 Overview of SECPI: explaining clustered process instances. If a certain feature (e.g. a certain relationship between activities B and E) had been different, the process instance would have been classified in a different cluster.

Our approach starts by projecting process instances onto feature vectors, like the white box, global classification model learning techniques as discussed above. To configure this featurisation phase, users can select one or more attribute templates to construct the binary feature vectors (for example: a template which generates a "presence" attribute for each activity in the log activity alphabet, denoting whether this activity occurs or not in the instance). Five control-flow features are presented in Table 3, which can be used in this regards. They are based on existing patterns from the process mining domain: the directly follows relation, notably used in the $\alpha$-algorithm (van der Aalst et al, 2004) and the always and sometimes follows relations, as described in Rozinat and van der Aalst (2008).

Four remarks should be made to this regard. First, note that users are not limited to the use of control-flow based attribute templates, but can also utilise or add custom templates incorporating other case data. As many currently existing clustering algorithms are control-flow driven, we have implemented five distinct control-flow attribute templates, as shown in Table 3 . Potentially in- 
Table 3 Control-flow attributes included in the current implementation

\begin{tabular}{|c|c|c|}
\hline Shorthand & Attribute Name & Explanation \\
\hline EXI & $\operatorname{Exists}(a)$ & Activity $a$ is present somewhere in the trace \\
\hline$A D F$ & AlwaysDirectlyFollows $(a, b)$ & $\begin{array}{l}\text { Activities } a \text { and } b \text { are present in the trace } \\
\text { and } a \text { is always directly followed by } b\end{array}$ \\
\hline$S D F$ & SometimesDirectlyFollows $(a, b)$ & $\begin{array}{l}\text { Activities } a \text { and } b \text { are present in the trace } \\
\text { and } a \text { is directly followed by } b \text { at least once }\end{array}$ \\
\hline$A W F$ & AlwaysWeaklyFollows $(a, b)$ & $\begin{array}{l}\text { Activities } a \text { and } b \text { are present in the trace } \\
\text { and } a \text { is always eventually followed by } b\end{array}$ \\
\hline$S W F$ & SometimesWeaklyFollows $(a, b)$ & $\begin{array}{l}\text { Activities } a \text { and } b \text { are present in the trace } \\
\text { and } a \text { is eventually followed by } b \text { at least once }\end{array}$ \\
\hline
\end{tabular}

teresting alternatives control-flow based attributes could be Declare constraints (Pesic et al, 2007), causal footprints (van Dongen et al, 2008) and behavioural profiles (Weidlich et al, 2011). Observe that a relationship already exists between the currently included attributes and the Declare language: Exists $(a)$ and AlwaysWeaklyFollows $(a, b)$ are the equivalent of Existence $(1, a)$ and $\operatorname{Response}(a, b)$ from the Declare language, for example. One could opt to specifically use noncontrol flow based attributes, such as other data related to the process instances. Secondly, observe that the incorporation of specific attributes inherently leads to a representational bias: it is likely that features used for the explanations are different than the ones used by the clustering algorithm. Such an approach is supported by Ribeiro et al (2016), in which the authors state that 'interpretable' explanations need to use a representation that is understandable to humans, regardless of the actual features used by the model.' This means that representational bias can be a good thing as long as it leads to interpretable explanations. As a third remark, it should be noted that the use of multiple or complex attribute templates may lead to massive dimensionality of the constructed data set. Therefore, we employ the well-known liblinear library for large-scale linear classification based on linear kernel SVMs supporting data with millions of instances and features (Fan et al, 2008). Observe that one could limit the generation of certain attributes that relate to log elements which are deemed less important, for instance by skipping SometimesDirectlyFollows $(a, b)$ attributes which relate to low-frequent activities, as it can then be assumed that the explanatory power of these attributes will be rather low. Finally, any constructed data set is likely to contain a large number of redundant features, because of zero variance or perfect correlation 1 . From extensive experimental evaluation, it turned out that removing these redundant features upfront increases performance and solution stability.

Observe that SECPI is inspired by the work of Martens and Provost (2014), who put forward an approach for explaining text document classifications. In the context of document classification, one is often confronted with limited comprehensibility of the predictive model, even despite using so-called white box techniques such as decision trees or logistic regression, which is mainly due to the high dimensionality. Similarly, this high dimensionality comes into play when characterising process instances by means of binary vectors representing control-flow characteristics. The main contribution of SECPI lies in its adaptation of the idea in (Martens and Provost, 2014) to trace clustering, with three major modifications. First, since most clustering solutions contain more than two clusters, support for multi-class predictions is included. Second, the algorithm is configured to only retrieve explanations regarding behaviour that is present in a trace. These explanations will most likely be of higher relevance than explanations that would artificially induce

1 Consider Exists(a) in a case where each trace starts with activity $a$, then this feature would correspond to a column of ones in the constructed data set. Hence, it can contain no discriminating information and is a redundant feature. In the same data set, with activity $a$ at the start of each trace, Exists(b) and SometimesWeaklyFollows $(a, b)$ will be perfectly correlated, making one of these features redundant. 
behaviour. Finally, performance has been optimised in several ways, including the prevention of repeat checking of equal combinations, the implementation of an early pruning mechanism for cases in which only the shortest explanation is of interest, and the upfront removal of redundant features, as discussed earlier.

\subsection{SECPI Algorithm (Search for Explanations for Clusters of Process Instances)}

Algorithm 1 provides a formalised overview of the workings of the SECPI algorithm.

As inputs, an instance to be explained (a process trace in a cluster) is given, defined as a sequence of binary attributes (generated using the attribute templates as discussed above). Note that for increased performance, it is advised to reduce redundant features upfront, as discussed earlier. Next, a classifier is assumed to be trained over the data set which is able to, for a given feature vector, return a predicted class label and associated score (i.e. probability). Finally, three configuration options can be set to configure the algorithm: iterations denotes the depth to search for explanations for the given instance. Increasing this value increases the run time but leads to more (albeit longer) explanations. The zero_to_one parameter denotes whether 0 to 1 attribute value swaps should be allowed. Since the instance attributes denote characteristics of the instance which are present (such as the direct following of two activities, for instance), it is recommended to set this parameter to False, as explanations denoting that a trace would not appear in its cluster when it did not present a specific characteristic are generally easier to interpret than explanations denoting that a trace should have a certain characteristic (as the question is then asked where and how exactly this characteristic would manifest itself within the trace). Additionally, since the multitude of all attributes for a trace are set to 0 , the set of retrieved rules will be shorter and better fine-tuned to the actual behaviour as seen in the process instance. Finally, the early_stop parameter denotes whether or not to stop searching for longer explanations once an explanation has been found.

As output, a set of explanatory rules is returned, formalised as a set of sets of attribute indices. Each set of indices represents a candidate explanation, and should be interpreted as follows: "this process instance would leave its current cluster when all the following attributes would be inverted" - or, in case where zero_to_one is set to False: "when it would not exhibit the behaviour as represented by these attributes". To construct this set of explanations, the algorithm applies a heuristic, best-first search procedure with pruning. First, each candidate single attribute is evaluated (lines 7 to 15 ) to see whether rules composed of only one attribute can be found. If swapping an attribute's value does not lead to a class change, a combination of indices (in this case a single index) is added to $E$ to be expanded in the next step.

Next, a number of iterations is performed (lines 17 to 34 ) as set by the iterations parameter. These iterations are interrupted if the user is not interested in retrieving longer explanations once an explanation has been found (line 18). A best-first candidate selection from all currently available combinations to expand on is chosen, based on the classifier's scoring function (line 19). The goal is to first explore the set of attribute indices for which swapping their values moves the instance farthest away from its current class label (i.e. cluster). Expansions on this combination are created by creating a new set of combinations combos' by adding each allowed attribute to the set of combo (lines 21 to 26). Expansions which are equal to combo (i.e. the added attribute was already used in combo) or which are subsumed by an already existing explanation (the expansion contains all attribute indices of an existing explanation and thus adds no value) are not considered. Once all expansions are built, they are evaluated to see if they lead to a class change (lines 27 to 34). Expanded combinations are removed from $E$ to prevent them being chosen again in the next iteration (line 33).

As a classification model, we use a combination of $k$ (the number of clusters) SVM models to allow for multi-class classification with SVMs. To retrieve the predicted class label and score, we apply a winner-takes-all strategy as follows. An SVM model is built per cluster to predict whether an instance is in-cluster 
(label: 1) or out-of-cluster (label: 0). To predict the label and probability of an instance, the probability that the instance is out or in their respective cluster is evaluated for all SVMs (with probability $p_{k}$ if predicted in-cluster and $1-p_{k}$ if predicted out-of-cluster). The SVM model with the highest probability determines the label (and its corresponding probability). Note that other classifiers (such as decision tree or rule based classifiers) could, in theory, also be applied in the SECPI algorithm as long as a scoring function can be defined, and in fact could also return small-sized instance explanations - as is our goal - even although their model itself (in terms of number of rules or decision tree nodes for example) can still be large. However, the construction of such models becomes unwieldy when dealing with high dimensional data sets, so that SVMs remain a suitable classifier for use within our proposed technique.

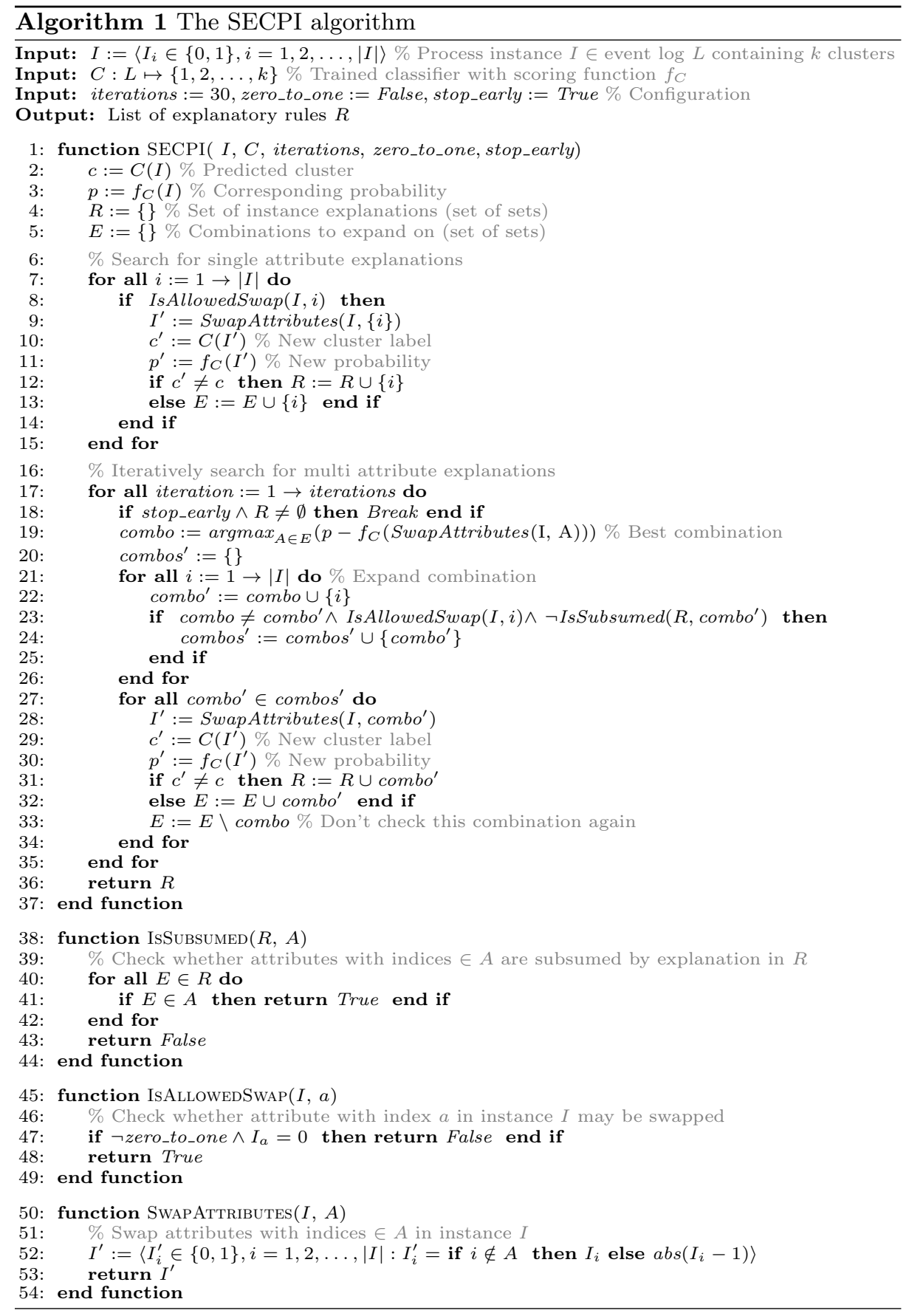




\subsection{Theoretical runtime complexity evaluation}

In this section, the theoretical runtime of the SECPI-algorithm is derived.

The complexity of creating the classification dataset depends on the number of features $|I|$. For example, if $a$ is the number of distinct activities present in the event $\log$, then there are $a$ possible features of the Exi kind, and $a^{2}$ of the others, as seen in Table 3. If there are $p$ process instances in the event log, creating an unpruned dataset with any feature other than Exi dataset takes $p * a^{2}$ steps of checking whether the attribute holds in a single process instance. In general, the steps needed for the creation of the dataset are of $O(p|I|)$ order.

As explained in Section 3.2, the next step of the algorithm is to train $k$ support vector machines, one for each cluster in the input dataset. The chosen solver is specifically known for its linear training time in terms of the number of instances (Fan et al, 2008), therefore this step has a complexity of $O(k p)$.

The remainder of the complexity can be derived from Algorithm1. Remark that the steps described in Algorithm 1 need to be performed for each process instance, thus $p$ times. From lines 7 to 15, each attribute is checked as an explanation by itself. In a worst-case scenario, no explanations are found: $R$ is empty and $|E|=|I|$. The complexity of this step is $O(|I|)$. From line 17 to 35 , the set of combinations to expand on, $E$, is iteratively expanded by taking the current best combination from the $|E|$ (line 19), which is done in $O(|E|)$, creating new combinations by expanding this best combination, in $O(|I|)$ (lines 21-26), and checking if an explanation has been found, in $O(|I|)$ (lines 27-34). In a worst case scenario, $|E|=|E|+|I|$ at the end of each iteration. Therefore, $|E|$ is always smaller than the number of iterations times $|I|$, and thus $O(|E|)=O(|I|)$. As such, we have shown that all steps in the third part of the Algorithm are $O(|I|)$ for a single process instance, or $O(p|I|)$ for all instances, the same complexity as creating the dataset. Combined with the complexity of training $k$ linear SVMs, the overall complexity is $O(p|I|+k p)$ or $O(p(|I|+k))$. In conclusion, our algorithm is linear in terms of process instances and number of clusters, and quadratic in terms of the number of distinct activities, unless the Exi feature is chosen.

\subsection{Aggregating instance level explanations using networked graphs}

While SECPI learns individual rules for each process instance, end users might be interested in an aggregated model-level interpretation of a clustering solution. As such, SECPI is extended with functionality that allows an end user to visualise distinct process instances in a networked graph based on a certain distance metric. Useful distance metrics in this case are for instance the number of shared rules or the ratio of shared attributes in the rules that explain process instances. By coloring traces according to their cluster membership, users can identify discrepancies and overlaps between clusters in terms of shared explanation characteristics.

Although a simple networked graph can already provide useful insights as is for instance demonstrated in the next section, this is only considered a first step towards providing human understanding at an aggregated level based on the SECPI technique. Future interesting leads include a more intuitive map-based visualisation of the clusters (Gansner et al, 2010), improved networked graphs for largescale visualisation (Abello et al, 2006), hybrid visualisation (Viau et al, 2010), and advanced visual linkage between visualisations (Collins and Carpendale, 2007).

\subsection{Implementation}

The technique is implemented in ProM ${ }^{2}$ an open source platform for process mining software, as the SVMExplainer plugin ${ }^{3}$. Apart from applying the SECPI algorithm, the end user has the option to interact with a visual inspector, allowing

\footnotetext{
2 http://www.promtools.org/prom6/

3 The plugin itself, screen captures and further explanation can be retrieved from: http://www.processmining.be/svmexplainer
} 


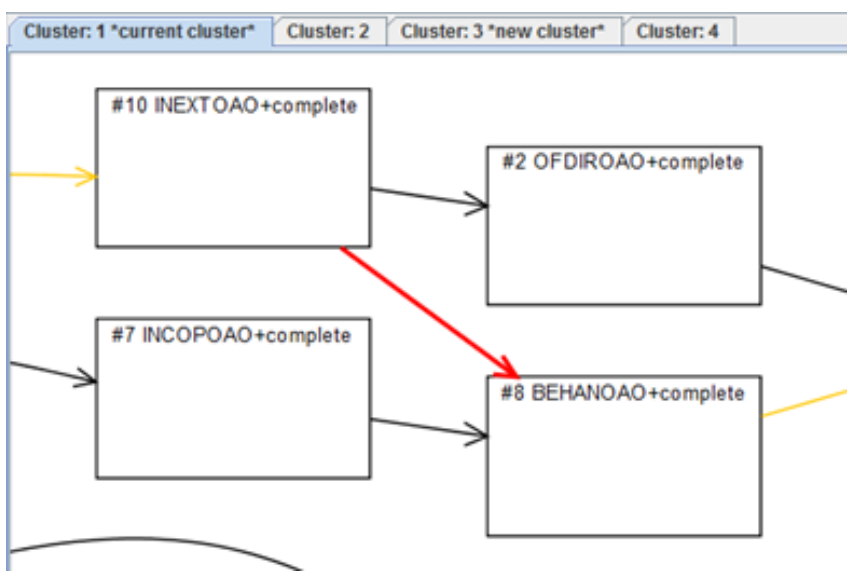

Fig. 3 Visualisation in the ProM-framework of the rule SomeTimesDirectlyFollows (Inextoao,Behanoao) in the tender dataset, clustered using ActFreq. There are 267 traces (of 2004 in total) that would have been predicted to be in cluster 3 instead of 1 had this relationship been false.

for the following analysis tasks. First, inspection of instances and their explanations (SECPI). Second, the projection of an instance and its explanation onto a visual (discovered) process model (built per cluster) to show where the characteristics contained in the explanation are located. Note that the feasibility of this projection depends on the attribute templates chosen and is therefore purely optional. As example, Figure 3 contains a visualisation on the tender data set, one of the datasets that is later used in the evaluation (Section 4). Attribute templates can be modified or added according to the requirements of analysts, even when no simple strategy to project them to a visual model exists. As a third analysis option, we have added the capability to aggregate instance level explanations ("show all instances which can be explained by this rule") in order to search for a minimal set of rules which explain all clustered instances. As such, a good middle ground technique is provided between very detailed instance-level based inspections and the large models given by global white box classifiers.

\section{Experimental Evaluation}

4.1 Comparison of SECPI and white box classification models

In order to compare SECPI to other approaches, an experimental evaluation is performed with 9 real-life logs (stemming from various sectors and workflow systems) and one educational artificial log (reviewing). All event logs have previously been used in experimental evaluations of process mining or trace clustering techniques (De Weerdt et al, 2012, 2013, Appice and Malerba, 2015). Table 4 provides an overview of the basic characteristics of the included logs, i.e. the number of instances, number of distinct instance variants, the number of activities and the average trace length in terms of events ${ }^{4}$

We evaluate the performance of SECPI in comparison with two white box global classifiers: RIPPER (Cohen, 1995) and C4.5 decision trees (Quinlan, 1993). In this section, we evaluate each technique using 4 clusters. The results of replications on logs clustered into 6 and 8 clusters are included in appendix, showing that similar results are found for other numbers of clusters. For creating the clusters, we apply 8 different algorithms as summarized in Table 7 Based on the clustered logs, we generate data sets using five different attribute templates as described in Table 3, which serve as a training set for each technique. Additionally, we have also added a configuration where all 5 of the attribute templates are combined. This configuration is denoted with $\mathrm{All}$.

4 The event logs are available for download on http://www.processmining.be/ svmexplainer/datasets 
Table 4 Event logs used in experimental setup with their characteristics

\begin{tabular}{|c|c|c|c|c|c|c|}
\hline Event Log & \#Instances & $\begin{array}{r}\text { \#Instance } \\
\text { Variants }\end{array}$ & \#Activities & $\begin{array}{r}\text { Average Trace Length } \\
\text { (\#Events) }\end{array}$ & & Source \\
\hline telecom & 17812 & 1908 & 42 & 4.68 & De Weerdt et al & $1(2012)$ \\
\hline purchase & 10487 & 76 & 23 & 9.33 & De Weerdt et al & $1,(2012)$ \\
\hline admin & 8384 & 277 & 163 & 6.17 & De Weerdt et al & $1(2012)$ \\
\hline insurance & 6407 & 155 & 18 & 6 & De Weerdt et al & $1(2012)$ \\
\hline tender & 2004 & 71 & 23 & 9.33 & De Weerdt et al & $1 \longdiv { ( 2 0 1 2 ) }$ \\
\hline incident & 1541 & 251 & 18 & 5.62 & De Weerdt et al & 12012 \\
\hline cProblem Volvo & 1487 & 327 & 7 & 4.48 & Steeman & 2013 \\
\hline environment & 1434 & 116 & 27 & 5.98 & Buijs & 2014 \\
\hline incman & 956 & 212 & 22 & 11.73 & De Weerdt et al & 12012 \\
\hline reviewing & 100 & 96 & 20 & 37.3 & van der Aalst & $(2016)$ \\
\hline
\end{tabular}

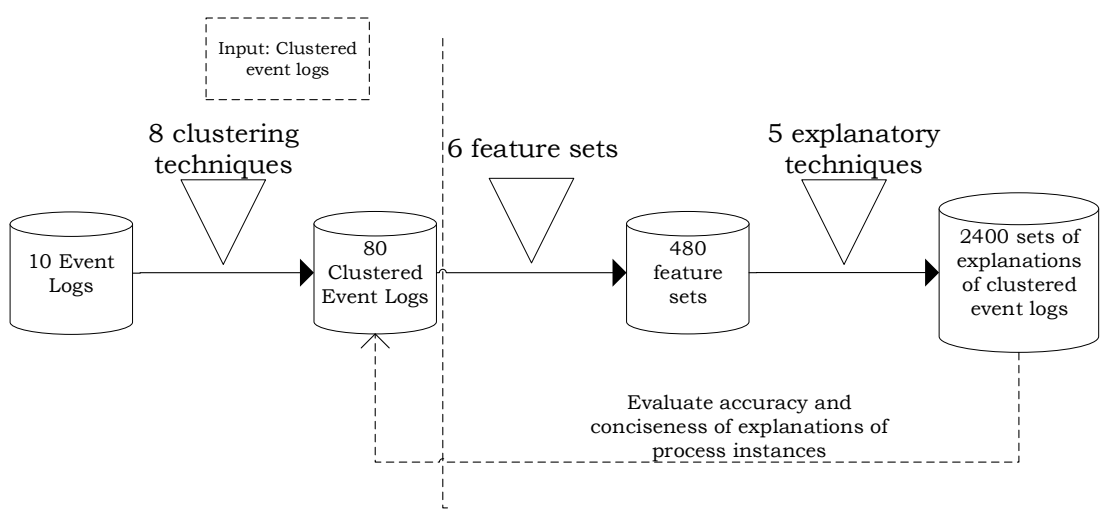

Fig. 4 Overview of the setup of the experiment

For RIPPER and C4.5, we mine both a model under the default parameters (with default pruning) and an adapted one. Default RIPPER in the Weka framework implies using three folds for reduced error pruning, a minimal weight of instances within a split of at least two, two optimization runs should be performed and a randomisation seed of one. For the adapted version, minimal required weight for splits is increased to $5 \%$ of the total amount of instances compared to default Ripper. It is clear that by imposing this restriction, accuracy will be traded for a smaller rule base. For C4.5, default settings imply a pruning confidence of 0.25 and at least two instances in each leaf. In the adapted setting, minimal leaf size is raised to $5 \%$ of the total amount of instances. The overall setup is summarized in Figure 4

Figure 5 (included here) and Figures 7 to 14 (included in Appendix) present a visualization of the results on each of the six event logs used. Two metrics are reported: on the one hand, classification accuracy is shown. This represents the fraction of instances the trained classifier (C4.5, RIPPER and the underlying multi-class SVM for SECPI) manages to classify in the right cluster. The plotted values represent averages over the 8 clustering techniques used. On the other hand, average explanation length is plotted. For SECPI, the length of the best explanation corresponds to the number of attributes incorporated in the shortest (i.e. first) explanation found ${ }^{5}$. For C4.5 and RIPPER, however, explanations for specific instances have to be derived in some other manner when using these classifiers as white box techniques. As such, for each instance, we investigate which path through the rule base or decision tree was followed in order to return a class label and derive the length from this explanation (i.e. the number of attributes involved in the conjunction to reach this outcome). Note that this alternative definition of

5 Consider for example the explanation "SometimesDirectlyFollows $(a, b)=0$ AND SometimesDirectlyFollows $(b, d)=0$ AND SometimesDirectlyFollows $(f, g)=0$ " denoting that this instance would leave the cluster if the three attributes corresponding to the sometimes directly follows relations listed above would be set to zero. The length of this explanation is thus equal to 3 . 

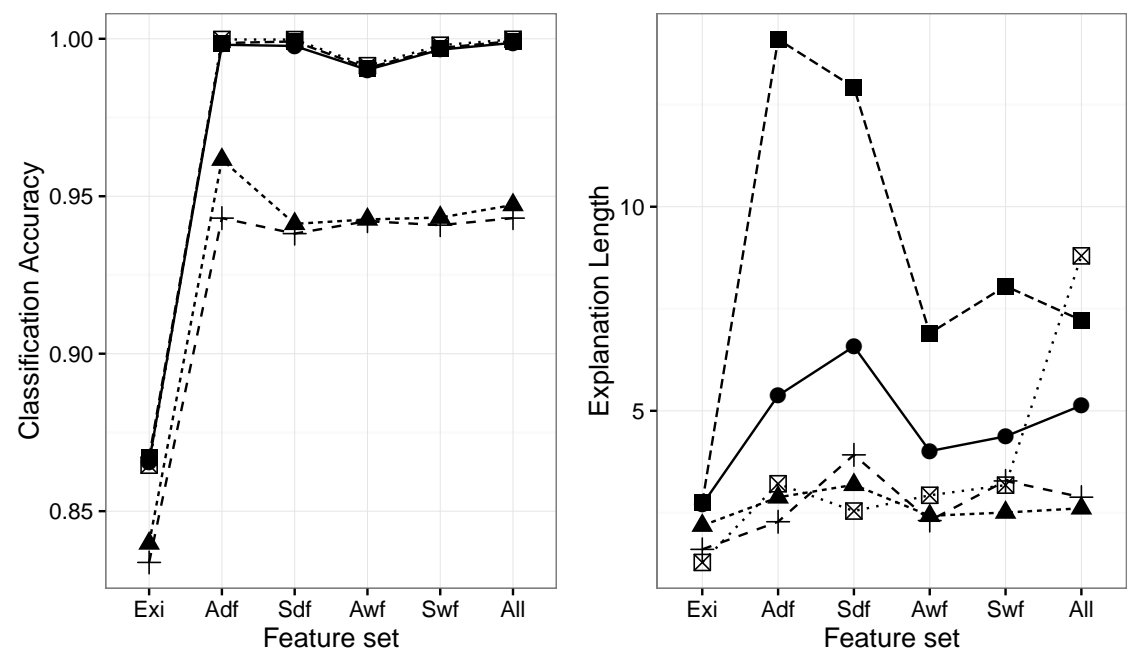

Technique $-\mathrm{C} 4.5$ (default)- $\mathbf{-} \cdot \mathrm{C} 4.5$ (min. leaf size)

Ripper (default)-+- Ripper (min. rule size) $\cdot$ 冈 SECPI

Fig. 5 Average accuracy (higher is better) and explanation length results (lower is better) for insurance $\log$

explanation length leads to a somewhat clouded comparison, as the explanations of the white-box classifiers are expected to be inherently more elaborate. There is no direct solution to this issue: if we would adapt RIPPER or C4.5 to contain the same concise information as SECPI, we would no longer be comparing SECPI based on support vector machines to off-the-shelf white-box-classifiers, but rather to SECPI itself, with C4.5 or RIPPER as underlying classifiers instead of support vector machines.

Table 5 contains results that are averaged over the clustered event logs. The reported accuracy is the result of a 10 -fold cross-validation. Since a support vector machine is trained for each cluster in SECPI, the cross-validated accuracy for SECPI is the average of the cross-validated accuracy of each of these support vector machines. Apart from the classification accuracy and explanation length, it also contains information on the average runtime of each combination (in seconds). The experiments were run on a Intel Xeon E5-2699 v3 processor of a Windows Server 2012 R2. There appears to be a distinct influence of the feature sets, as confirmed in the anaylsis of the theoretical runtime, Section 3.3. SECPI requires less runtime than default Ripper and C4.5 on average, supporting our choice for linear SVMs as an underlying classifier, as described in Section 3.1.

Although there does not exist a straightforward way to configure the derived balance between accuracy and explanation length, we are able to identify combinations of explanatory techniques and explanatory features that are Pareto efficient with regard to both measures for the tested event logs. This means that for these combinations, one cannot find a combination that improves one metric without worsening the other. Two such combinations can be found in Table 5 SECPI combined with EXI and Sdf. The classification accuracy and explanation length of these two combinations results is presented in boldface and underlined. Furthermore, combinations that are not significantly different from the Pareto-optimal combinations at a 5\% level regarding both accuracy and explanation length are included in boldface. For this significance, a one-tailed paired t-test is performed, where the pairing is based on the underlying clustering techniques and datasets. A Bonferroni correction for multiple comparisons is taken into account.

Furthermore, Table 5 contains a couple of technique-specific metrics. For the two algorithms based on decision trees, the average tree size and number of leaves are reported. The restricted version of C4.5 clearly results in smaller trees. For the two rule-based algorithms, the total number of rules in the rule base is listed. Lastly, the percentage of 'explainable' instances is listed for SECPI. With explainable, we refer to the number of instances that can be explained with the zero_to_one parameter set to False, given the maximum amount of iterations. As previously 
Table 5 Results of the experimental evaluation comparing SECPI with C4.5 and RIPPER averaged over clustering techniques and datasets, for a cluster number of 4

\begin{tabular}{|c|c|c|c|c|c|c|c|c|}
\hline 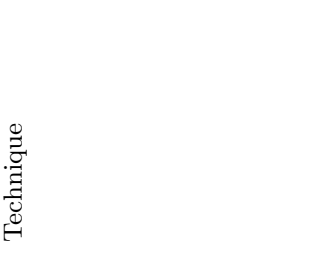 & 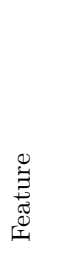 & 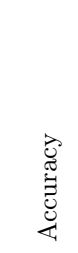 & 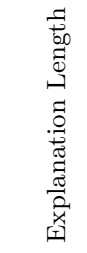 & 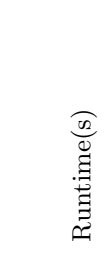 & 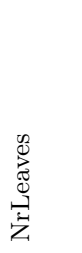 & 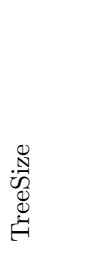 & 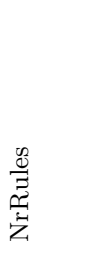 & 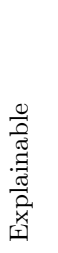 \\
\hline C4.5 (default) & Exi & 0.85 & 4.49 & 5.58 & 14.35 & 27.7 & - & - \\
\hline C4.5 (default) & $A d f$ & 0.94 & 6.33 & 95.84 & 28.62 & 56.25 & - & - \\
\hline C4.5 (default) & $S d f$ & 0.94 & 7.21 & 119.24 & 28.29 & 55.58 & - & - \\
\hline C4.5 (default) & $A w f$ & 0.93 & 5.40 & 127.13 & 18.74 & 36.48 & - & - \\
\hline C4.5 (default) & $S w f$ & 0.95 & 5.70 & 128.59 & 23.65 & 46.3 & - & - \\
\hline C4.5 (default) & All & 0.95 & 6.21 & 459.99 & 25.1 & 49.2 & - & - \\
\hline C4.5 (min. leaf size) & Exi & 0.83 & 2.71 & 2.41 & 3.36 & 5.72 & - & - \\
\hline C4.5 (min. leaf size) & $A d f$ & 0.88 & 2.93 & 29.34 & 3.86 & 6.72 & - & - \\
\hline C4.5 (min. leaf size) & $S d f$ & 0.87 & 2.99 & 27.96 & 3.81 & 6.62 & - & - \\
\hline C4.5 (min. leaf size) & $A w f$ & 0.88 & 2.89 & 45.25 & 3.67 & 6.35 & - & - \\
\hline C4.5 (min. leaf size) & $S w f$ & 0.90 & 2.95 & 46.17 & 3.88 & 6.75 & - & - \\
\hline C4.5 (min. leaf size) & All & 0.91 & 2.96 & 158.73 & 3.86 & 6.72 & - & - \\
\hline Ripper (default) & $E x i$ & 0.85 & 6.41 & 15.57 & - & - & 6.24 & - \\
\hline Ripper (default) & $A d f$ & 0.94 & 15.18 & 385.79 & - & - & 12.45 & - \\
\hline Ripper (default) & $S d f$ & 0.94 & 15.40 & 452.28 & - & - & 14.41 & - \\
\hline Ripper (default) & $A w f$ & 0.92 & 11.11 & 403.30 & - & - & 9.05 & - \\
\hline Ripper (default) & $S w f$ & 0.94 & 14.07 & 533.57 & - & - & 10.86 & - \\
\hline Ripper (default) & All & 0.95 & 14.71 & 1680.20 & - & - & 11.26 & - \\
\hline Ripper (min. rule weight) & Exi & 0.80 & 1.86 & 3.66 & - & - & 2.14 & - \\
\hline Ripper (min. rule weight) & $A d f$ & 0.85 & 2.47 & 54.00 & - & - & 2.5 & - \\
\hline Ripper (min. rule weight) & $S d f$ & 0.84 & 3.01 & 57.93 & - & - & 2.52 & - \\
\hline Ripper (min. rule weight) & $A w f$ & 0.85 & 2.51 & 93.21 & - & - & 2.45 & - \\
\hline Ripper (min. rule weight) & $S w f$ & 0.86 & 3.22 & 104.42 & - & - & 2.61 & - \\
\hline Ripper (min. rule weight) & All & 0.87 & 2.90 & 304.26 & - & - & 2.64 & - \\
\hline SECPI & Exi & $\underline{0.94}$ & $\underline{1.56}$ & 6.34 & - & - & - & 0.81 \\
\hline SECPI & $A d f$ & 0.98 & 2.69 & 10.96 & - & - & - & 0.96 \\
\hline SECPI & $S d f$ & $\underline{0.98}$ & $\underline{2.45}$ & 7.09 & - & - & - & 0.93 \\
\hline SECPI & $A w f$ & 0.97 & 3.20 & 23.96 & - & - & - & 0.92 \\
\hline SECPI & $S w f$ & 0.98 & 3.82 & 21.45 & - & - & - & 0.93 \\
\hline SECPI & All & 0.98 & 8.38 & 84.72 & - & - & - & 0.97 \\
\hline
\end{tabular}

explained, we assume that explanations using behaviour that was present in the process instance are more preferable to an end user than explanations that introduce artificial features. 'Explainable' instances are instances for which such an explanation can be extracted from the underlying SVMs. Clearly, this value is bounded by the percentage of instances that can be accurately classified by the SVMs. Dropping this restriction by changing the zero_to_one parameter to True leads to the results in Table 6. Ceteris paribus, explanations become $32 \%$ shorter on average, with no changes in terms of accuracy. Furthermore, the increased search space causes the runtime to inflate with an average factor of 34 , all other things equal.

A couple of observations can be made regarding Table 5 . First, it is clear that both classification accuracy and explanation length are influenced by the explanatory technique and the chosen feature set. With regards to accuracy, default C4.5, default Ripper, and SECPI perform similarly, clearly outperforming the restricted versions of $\mathrm{C} 4.5$ and Ripper. Of the different feature sets All performs best, as expected, since it is the combination of all the separate feature sets. Furthermore, explanatory techniques using Exi-features score distinctly worse on accuracy. SECPI's results combine better accuracy than the default white-box classifiers with shorter explanations than the restricted ones when combined with datasets based on Exi or Sdf. Furthermore, an extra note should be made with regards to SECPI combined with the complete feature set All: it leads to oversized 
Table 6 Experimental results of SECPI on varying clustering and features, averaged over the datasets with zero_to_one set to True

\begin{tabular}{|c|c|c|c|c|c|}
\hline 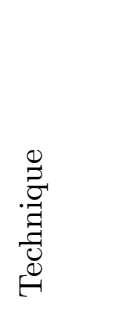 & 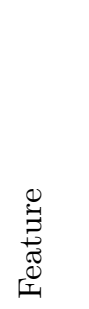 & 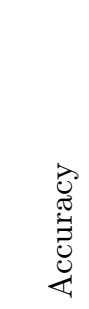 & 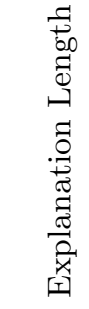 & 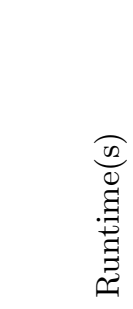 & 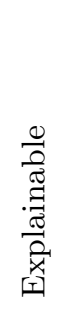 \\
\hline SECPI & Exi & 0.94 & 1.22 & 26.61 & 0.86 \\
\hline SECPI & $A d f$ & 0.98 & 1.78 & 371.11 & 0.98 \\
\hline SECPI & $S d f$ & 0.98 & 1.50 & 295.79 & 0.97 \\
\hline SECPI & $A w f$ & 0.97 & 1.97 & 622.30 & 0.95 \\
\hline SECPI & $S w f$ & 0.98 & 1.97 & 718.66 & 0.97 \\
\hline SECPI & All & 0.98 & 3.95 & 4746.69 & 0.99 \\
\hline
\end{tabular}

explanations. A possible explanation for this phenomenon lies with the inherent correlation between many of its feature vectors, and is an interesting lead for future research. An example could be looking into training sparser underlying support vector machines or performing stricter feature selection. Finally, the results of a replication of our experiments on the same logs clustered into 6 and 8 clusters instead of 4 are provided in appendix as Table 9 and 10. The results in terms of accuracy and explanation length are similar to those in Table 5 . Therefore, our observations remain valid for other numbers of clusters.

\subsection{Utilizing SECPI to compare clustering solutions}

In this section, we give an example of how SECPI can be deployed to facilitate the choice between different trace clustering techniques.

Table 7 Clustering techniques included in experimental evaluation

\begin{tabular}{lll}
\hline Shorthand & Technique & Implementation \\
\hline ActFreq & Frequency-based ActiTrac & ActiTrac-plugin (ProM 6) \\
ActMRA & Distance-based ActiTrac & ActiTrac-plugin (ProM 6) \\
$M R$ & AHC based on Maximal Repeat & GuideTreeMiner-plugin (ProM 6) \\
$M R A$ & AHC based on Maximal Repeat Alphabet & GuideTreeMiner-plugin (ProM 6) \\
GED & AHC based on Generic Edit Distance & GuideTreeMiner-plugin (ProM 6) \\
LED & AHC based on Levenshtein Edit Distance & GuideTreeMiner-plugin (ProM 6) \\
BOA & AHC based on Bags of Activities & GuideTreeMiner-plugin (ProM 6) \\
3-gram & AHC based on $k$-grams & GuideTreeMiner-plugin (ProM 6) \\
\hline Note & AHC: Agglomerative Hierarchical Clustering & \\
\hline
\end{tabular}

To provide the reader with a sense of the relationships between the different clustering techniques, Figure 6 contains a visualisation of the similarity of the solutions of the 8 different clustering techniques presented in Table 7. For this visualisation, the Normalized Mutual Information (NMI, Fred and Lourenço (2008)) shared by two clustering solutions is used, where $L$ is a set of event logs and $P^{a}$ and $P^{b}$ are two clustering solutions:

$$
\operatorname{aNMI}\left(P^{a}, P^{b}\right)=\frac{1}{|L|} \sum_{l \in L}\left(N M I\left(P^{a}, P^{b}\right)\right)
$$

In this specific case, $L$ is the set of 10 event logs as shown in 4 . From Figure 6, we can see that most of the algorithms based on agglomerative hierarchical clustering 


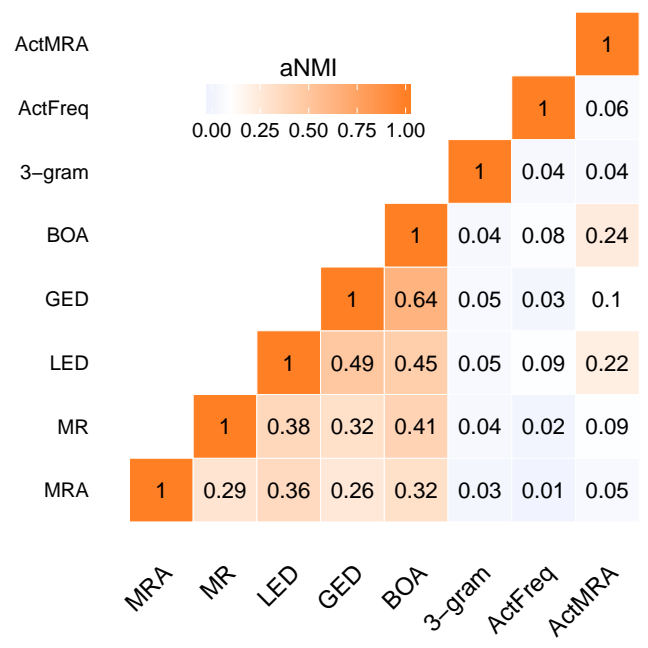

Fig. 6 Visualising similarity in cluster solutions using a heat map

produce similar results, except for 3-gram. ActMRA, a variant of ActFreq that incorporates a similar measure as the $M R A$, appears to partly contain similar information as BOA and LED. ActFreq and 3-gram result in reasonably unique clustering solutions.

In this section we will employ the environment event log, as an example. Table 4 contains some general statistics on this log. Given that different clustering techniques lead to different clustering results, the SECPI-algorithm can be used to decide which technique is appropriate for a given dataset. In Table 8 , the results of using SECPI to explain different clustered event logs are depicted. SECPI is given AlwaysDirectlyFollows-attributes to create explanations. A couple of observations can be made from these results: first, note that the accuracy is almost equal in each of the combinations. Nonetheless, there appears to be a difference in terms of explainability: the clustered logs created by the 3-gram and $M R A$ techniques can not be explained fully. Recall that this is likely due to the zero_to_one parameter which is set to false: simple, non-behaviour inducing explanations are favoured. The final observation is one in terms of explanation length: ceteris paribus, a clustering solution that is easy to understand is preferable. In this regard, the cluster event solutions created with the $G E D$ and $M R$ techniques are superior.

The previous section contained an illustration of how SECPI can be used to support the learning phase of a clustering exercise: its results can be used to decide which clustering technique is appropriate. However, it is advisory to take other dimensions into account when selecting a clustering technique, as SECPI is inherently restricted to the representational bias of the selected features, and understandability is only one dimension of importance.

\section{Conclusion}

In this paper, SECPI (Search for Explanations of Clusters of Process Instances), a new technique for providing human understanding for trace clustering results was presented. The need for such a technique stems from the observation that typical trace clustering techniques do not provide sufficient insight into how a clustering solution is composed. Furthermore, it was argued that various potential alternative techniques, for instance a visual comparative analysis of the underlying discovered process models, fall short in resolving the problem at hand. Accordingly, our SECPI-algorithm aims to find a minimal set of control-flow-based characteristics such that - if these characteristics were not present - the instance would not remain in its current cluster. In this way, the technique is capable of discerning concise, individual rules that clearly explain why a certain instance is part of a cluster. In addition, its implementation as a ProM plugin allows for visualising an explanation in the respective discovered process models underlying the clusters. 
Table 8 Results of using SECPI with the AlwaysDirectlyFollows feature on the environment event log clustered with varying clustering techniques at a number of clusters of 4 , and zero_to_one set to False.

\begin{tabular}{|c|c|c|c|c|c|}
\hline 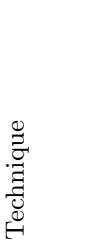 & 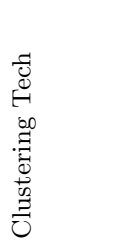 & 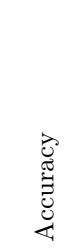 & 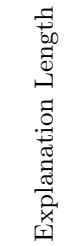 & 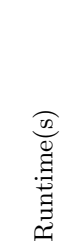 & 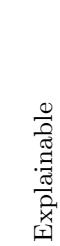 \\
\hline SECPI & ActFreq & 0.99 & 1.73 & 2.44 & 1.00 \\
\hline SECPI & ActMRA & 0.99 & 2.29 & 1.30 & 1.00 \\
\hline SECPI & 3-gram & 1.00 & 4.59 & 1.51 & 0.92 \\
\hline SECPI & $B O A$ & 0.99 & 1.76 & 2.60 & 1.00 \\
\hline SECPI) & $G E D$ & 0.99 & 1.49 & 2.27 & 1.00 \\
\hline SECPI & $L E D$ & 1.00 & 2.24 & 1.10 & 1.00 \\
\hline SECPI & $M R$ & 0.99 & 1.59 & 2.36 & 1.00 \\
\hline SECPI & $M R A$ & 1.00 & 1.92 & 2.54 & 0.92 \\
\hline
\end{tabular}

In the experimental evaluation, it was shown that, combined with certain rule features, SECPI is able to strike a better balance between accuracy and comprehensibility as compared to typical white box classification techniques such as rule and decision tree learners. Furthermore, it was shown that the SECPI algorithm and its implementation can enable a more well-founded choice of trace clustering technique.

In future work, we foresee to expand on a number of closely related topics. First, we plan to investigate the incorporation of additional domain knowledge using non control-flow-based attributes. Potential routes include the resource and performance perspectives proposed in (Appice and Malerba, 2015). Second, aggregation of instance-level explanations is a worthwhile research track as well. As explained in Section 3.4 the current implementation already supports the investigation of shared explanations amongst groups of instances. In the future, we plan to make the global versus instance-based trade-off more configurable towards the end user. Thirdly, we aim to investigate the effects of the inherent multicollinearity of the feature vectors on the performance of the underlying support vector machines. While accuracy remains stable, average explanation length was shown to be quite sensitive to these effects. Finally, future research will focus on the practical applicability of SECPI. It is argued that the technique is ideally suited to relate exogenously defined clusters, e.g. high versus low cost instances, to process-specific control-flow characteristics.

\section{References}

Abello J, van Ham F, Neeraj Krishnan (2006) ASK-GraphView: A Large Scale Graph Visualization System. IEEE Trans Vis Comput Graph 12(5):669-676, doi: 10.1109 /TVCG.2006.120

Adriansyah A, van Dongen BF, van der Aalst WMP (2011) Conformance checking using cost-based fitness analysis. In: EDOC, pp 55-64, doi: $10.1109 /$ EDOC.2011.12

Andrews R, Diederich J, Tickle AB (1995) Survey and critique of techniques for extracting rules from trained artificial neural networks. Knowledge-based systems 8(6):373-389

Appice A, Malerba D (2015) A co-training strategy for multiple view clustering in process mining. IEEE Transactions on Services Computing PP(99):1-1, doi: 10.1109 /TSC.2015.2430327

Bose RPJC, van der Aalst WMP (2009) Context aware trace clustering: Towards improving process mining results. In: SDM, pp 401-412, doi $10.1137 / 1.9781611972795 .35$ 
Bose RPJC, van der Aalst WMP (2010) Trace clustering based on conserved patterns: Towards achieving better process models. In: Lect. Notes Bus. Inf. Process., vol 43 LNBIP, pp 170-181, doi:10.1007/978-3-642-12186-9_16

Buijs J (2014) Environmental permit application process (wabo), coselog project. Eindhoven University of Technology, Dataset, doi:10.4121/uuid:26aba40d-8b2d435b-b5af-6d4bfbd7a270

Cadez I, Heckerman D, Meek C, Smyth P, White S (2003) Model-based clustering and visualization of navigation patterns on a web site. Data Mining and Knowledge Discovery 7(4):399-424, doi:10.1023/A:1024992613384

Chesani F, Lamma E, Mello P, Montali M, Riguzzi F, Storari S (2009) Exploiting inductive logic programming techniques for declarative process mining. In: Jensen K, van der Aalst WMP (eds) Transactions on Petri Nets and Other Models of Concurrency II: Special Issue on Concurrency in Process-Aware Information Systems, Springer Berlin Heidelberg, pp 278-295, doi:10.1007/978-3642-00899-3_16

Cohen W (1995) Fast effective rule induction. In: Prieditis A, Russell S (eds) Proceedings of the 12th International Conference on Machine Learning, Morgan Kaufmann Publishers, Tahoe City, CA, pp 115-123

Collins C, Carpendale S (2007) VisLink: Revealing relationships amongst visualizations. IEEE Trans Vis Comput Graph 13(6):1192-1199, doi:10.1109/TVCG.2007.70521

Cook JE, Wolf AL (1998) Discovering models of software processes from eventbased data. ACM Transactions on Software Engineering and Methodology $7(3): 215-249$

de Medeiros AKA, Weijters AJMM, van der Aalst WMP (2007) Genetic process mining: An experimental evaluation. Data Min Knowl Discov 14(2):245-304, doi:10.1007/s10618-006-0061-7

de Medeiros AKA, van der Aalst WMP, Weijters AJMM (2008) Quantifying process equivalence based on observed behavior. Data Knowl Eng 64(1):55-74, doi $10.1016 /$ j.datak.2007.06.010

De Weerdt J, Vanden Broucke S (2014) SECPI: Searching for explanations for clustered process instances. In: Lect. Notes Comput. Sci. (including Subser. Lect. Notes Artif. Intell. Lect. Notes Bioinformatics), vol 8659 LNCS, pp 408415, doi:10.1007/978-3-319-10172-9_29

De Weerdt J, De Backer M, Vanthienen J, Baesens B (2012) A multi-dimensional quality assessment of state-of-the-art process discovery algorithms using real-life event logs. Inf Syst 37(7):654-676, doi $10.1016 /$ j.is.2012.02.004

De Weerdt J, Vanden Broucke S, Vanthienen J, Baesens B (2013) Active trace clustering for improved process discovery. IEEE Trans Knowl Data Eng 25(12):27082720, doi $10.1109 /$ TKDE.2013.64

Delias P, Doumpos M, Grigoroudis E, Manolitzas P, Matsatsinis N (2015) Supporting healthcare management decisions via robust clustering of event logs. Knowledge-Based Syst 84:203-213, doi $10.1016 /$ j.knosys.2015.04.012

Dijkman R, Dumas M, Van Dongen B, Krik R, Mendling J (2011) Similarity of business process models: Metrics and evaluation. Inf Syst 36(2):498-516, doi $10.1016 /$ j.is.2010.09.006

Dijkman RM (2007) A classification of differences between similar business processes. In: EDOC, pp 37-50, doi 10.1109/EDOC.2007.24

Dijkman RM (2008) Diagnosing differences between business process models. In: BPM, pp 261-277, doi $10.1007 / 978-3-540-85758-7 \_20$

van Dongen BF, Dijkman RM, Mendling J (2008) Measuring similarity between business process models. In: CAiSE, pp 450-464, doi:10.1007/978-3-540-695349_34

Dumas M, La Rosa M, Mendling J, Reijers HA (2013) Fundamentals of Business Process Management. Springer, doi:10.1007/978-3-642-33143-5

Ekanayake CC, Dumas M, García-Bañuelos L, La Rosa M (2013) Slice, mine and dice: Complexity-aware automated discovery of business process models. In: BPM, pp 49-64, doi 10.1007/978-3-642-40176-3_6

Evermann J, Thaler T, Fettke P (2016) Clustering traces using sequence alignment. In: Reichert M, Reijers HA (eds) Business Process Management Workshops: BPM 2015, 13th International Workshops, Innsbruck, Austria, August 31 
- September 3, 2015, Revised Papers, Springer International Publishing, Cham, pp 179-190, doi:10.1007/978-3-319-42887-1_15

Fan RE, Chang KW, Hsieh CJ, Wang XR, Lin CJ (2008) Liblinear: A library for large linear classification. The Journal of Machine Learning Research 9:18711874, doi 10.1038/oby.2011.351

Fayyad U, Piatetsky-Shapiro G, Smyth P (1996) Knowledge Discovery and Data Mining: Towards a Unifying Framework. Proc 2nd Int Conf Knowl Discov Data Min Portl OR pp 82-88

Ferreira DR, Zacarias M, Malheiros M, Ferreira P (2007) Approaching process mining with sequence clustering: Experiments and findings. In: BPM, pp 360374, doi 10.1007/978-3-540-75183-0_26

Fisher DH (1987) Knowledge acquisition via incremental conceptual clustering. Machine learning 2(2):139-172

Folino F, Greco G, Guzzo A, Pontieri L (2011) Mining usage scenarios in business processes: Outlier-aware discovery and run-time prediction. Data Knowl Eng 70(12):1005-1029, doi:10.1016/j.datak.2011.07.002

Fred A, Lourenço A (2008) Cluster ensemble methods: from single clusterings to combined solutions. In: Supervised and unsupervised ensemble methods and their applications, Springer, pp 3-30, doi:10.1007/978-3-540-78981-9_1

Gansner ER, Hu Y, Kobourov S (2010) Visualizing graphs and clusters as maps. IEEE Comput Graph Appl 30(6):54-66, doi 10.1109/MCG.2010.101

van Glabbeek RJ, Goltz U (2001) Refinement of actions and equivalence notions for concurrent systems. Acta Inf 37(4/5):229-327, doi:10.1007/s002360000041

Goedertier S, Martens D, Vanthienen J, Baesens B (2009) Robust process discovery with artificial negative events. J Mach Learn Res 10:1305-1340, doi $10.1145 / 1577069.1577113$

Greco G, Guzzo A, Pontieri L, Saccà D (2006) Discovering expressive process models by clustering log traces. IEEE Trans Knowl Data Eng 18(8):1010-1027, doi:10.1109/TKDE.2006.123

Günther CW, Verbeek H (2014) Xes-standard definition. BPM Center Report BPM-14-09, BPMcenterorg

Hidders J, Dumas M, van der Aalst WMP, ter Hofstede AHM, Verelst J (2005) When are two workflows the same? In: Proceedings of the 2005 Australasian Symposium on Theory of Computing - Volume 41, Australian Computer Society, Inc., Darlinghurst, Australia, Australia, CATS '05, pp 3-11, URL http://dl. acm.org/citation. cfm?id=1082260.1082261

Kiepuszewski B, ter Hofstede AHM, van der Aalst WMP (2003) Fundamentals of control flow in workflows. Acta Inf 39(3):143-209, doi:10.1007/s00236-002-01054

Lamma E, Mello P, Riguzzi F, Storari S (2008) Applying inductive logic programming to process mining. In: Blockeel H, Ramon J, Shavlik J, Tadepalli P (eds) Inductive Logic Programming: 17th International Conference, ILP 2007, Corvallis, OR, USA, June 19-21, 2007, Revised Selected Papers, Springer Berlin Heidelberg, Berlin, Heidelberg, pp 132-146, doi:10.1007/978-3-540-78469-2_16

Levenshtein VI (1966) Binary Codes Capable of Correcting Deletions, Insertions and Reversals. Soviet Physics Doklady 10:707-710

Martens D, Provost F (2014) Explaining Data-Driven Document Classifications. MIS Q 38(1):73-99

Martens D, Baesens B, Gestel TV, Vanthienen J (2007) Comprehensible credit scoring models using rule extraction from support vector machines. European Journal of Operational Research 183(3):1466 - 1476, doi:10.1016/j.ejor.2006.04.051

Michalski RS, Stepp RE (1983) Learning from observation: Conceptual clustering. In: Machine learning, Springer, pp 331-363

Mitchell TM, Keller RM, Kedar-Cabelli ST (1986) Explanationbased generalization: A unifying view. Machine Learning 1(1):47-80, doi $10.1023 / \mathrm{A}: 1022691120807$

Pesic M, Schonenberg H, van der Aalst WM (2007) Declare: Full support for loosely-structured processes. In: Enterprise Distributed Object Computing Conference, 2007. EDOC 2007. 11th IEEE International, IEEE, pp 287-287, doi:10.1109/EDOC.2007.14 
Quinlan J (1993) C4.5: programs for machine learning. Morgan Kaufmann Publishers Inc., San Francisco, CA, USA

Ribeiro MT, Singh S, Guestrin C (2016) "why should i trust you?": Explaining the predictions of any classifier. In: Proceedings of the 22Nd ACM SIGKDD International Conference on Knowledge Discovery and Data Mining, ACM, New York, NY, USA, KDD '16, pp 1135-1144, doi:10.1145/2939672.2939778

Rozinat A, van der Aalst WMP (2006) Decision mining in ProM. In: Business Process Management, pp 420-425, doi:10.1007/11841760_33

Rozinat A, van der Aalst WMP (2008) Conformance checking of processes based on monitoring real behavior. Information Systems 33(1):64-95, doi: $10.1016 /$ j.is.2007.07.001

Sole M, Carmona J (2011) Region-based foldings in process discovery. IEEE Trans Knowl Data Eng 25(1):192-205, doi 10.1109/TKDE.2011.192

Song M, Günther CW, van der Aalst WMP (2008) Trace clustering in process mining. In: BPM Workshops, pp 109-120, doi:10.1007/978-3-642-00328-8_11

Song M, Yang H, Siadat SH, Pechenizkiy M (2013) A comparative study of dimensionality reduction techniques to enhance trace clustering performances. Expert Syst Appl 40:3722-3737, doi:10.1016/j.eswa.2012.12.078

Steeman W (2013) Bpi challenge 2013. Ghent University. Dataset, doi $10.4121 /$ uuid:a7ce5c55-03a7-4583-b855-98b86e1a2b07

van der Aalst WMP (1999) Formalization and verification of eventdriven process chains. Information and Software technology 41(10):639-650, doi: 10.1016/S0950-5849(99)00016-6

van der Aalst WMP (2016) Process Mining - Data Science in Action, 2nd edn. Springer-Verlag Berlin Heidelberg, doi:10.1007/978-3-662-49851-4

van der Aalst WMP, Weijters T, Maruster L (2004) Workflow mining: Discovering process models from event logs. IEEE Trans Knowl Data Eng 16(9):1128-1142, doi: $10.1109 /$ TKDE.2004.47

van der Aalst WMP, de Medeiros AKA, Weijters AJMM (2006) Process equivalence: Comparing two process models based on observed behavior. In: Business Process Management, pp 129-144, doi:10.1007/11841760_10

Veiga GM, Ferreira DR (2010) Understanding spaghetti models with sequence clustering for prom. In: Rinderle-Ma, S et al (ed) BPM Workshops, Springer, LNBIP, vol 43, pp 92-103, doi:10.1007/978-3-642-12186-9

Viau C, McGuffin MJ, Chiricota Y, Jurisica I (2010) The FlowVizMenu and parallel scatterplot matrix: Hybrid multidimensional visualizations for network exploration. IEEE Trans Vis Comput Graph 16(6):1100-1108, doi:10.1109/TVCG.2010.205

Wang F, Sun J (2014) Survey on distance metric learning and dimensionality reduction in data mining. Data Min Knowl Discov 29(2):534-564, doi $10.1007 / \mathrm{s} 10618-014-0356-\mathrm{z}$

Weidlich M, Mendling J, Weske M (2011) Efficient consistency measurement based on behavioral profiles of process models. IEEE Trans Software Eng 37(3):410429, doi 10.1109 /TSE.2010.96

Weijters AJMM, van der Aalst WMP, Alves de Medeiros AK (2006) Process mining with the heuristicsminer algorithm. BETA working paper series 166, TU Eindhoven 
Appendix

Table 9 Results of the experimental evaluation comparing SECPI with C4.5 and RIPPER averaged over clustering techniques and datasets, replicated with a cluster number of 6

\begin{tabular}{|c|c|c|c|c|c|c|c|c|}
\hline 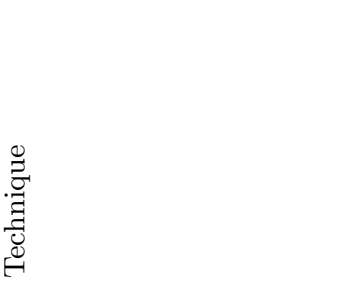 & 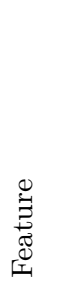 & 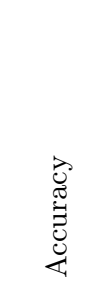 & 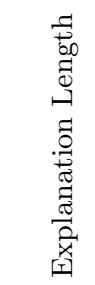 & 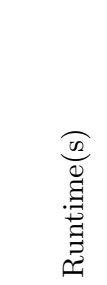 & 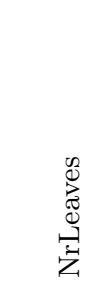 & 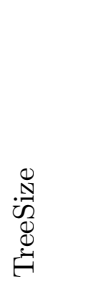 & 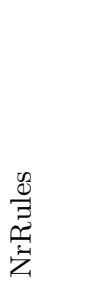 & 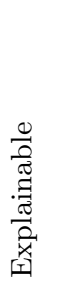 \\
\hline C4.5 (default) & Exi & 0.78 & 4.68 & 5.22 & 8.72 & 16.45 & - & - \\
\hline C4.5 (default) & $A d f$ & 0.88 & 6.83 & 31.34 & 19.95 & 38.9 & - & - \\
\hline C4.5 (default) & $S d f$ & 0.88 & 8.10 & 33.88 & 24.7 & 48.4 & - & - \\
\hline C4.5 (default) & $A w f$ & 0.85 & 6.30 & 33.81 & 15.28 & 29.55 & - & - \\
\hline C4.5 (default) & $S w f$ & 0.89 & 6.45 & 33.69 & 18.88 & 36.75 & - & - \\
\hline C4.5 (default) & All & 0.90 & 7.07 & 113.09 & 21.4 & 41.8 & - & - \\
\hline C4.5 (min. leaf size) & Exi & 0.75 & 2.98 & 2.40 & 4 & 7 & - & - \\
\hline C4.5 (min. leaf size) & $A d f$ & 0.80 & 3.68 & 13.26 & 5.6 & 10.2 & - & - \\
\hline C4.5 (min. leaf size) & $S d f$ & 0.78 & 3.47 & 11.93 & 5.03 & 9.05 & - & - \\
\hline C4.5 (min. leaf size) & $A w f$ & 0.79 & 3.61 & 15.50 & 5.33 & 9.65 & - & - \\
\hline C4.5 (min. leaf size) & $S w f$ & 0.83 & 3.51 & 15.61 & 5.35 & 9.7 & - & - \\
\hline C4.5 (min. leaf size) & All & 0.84 & 3.72 & 55.13 & 5.38 & 9.75 & - & - \\
\hline Ripper (default) & Exi & 0.76 & 5.75 & 11.82 & - & - & 6.15 & - \\
\hline Ripper (default) & $A d f$ & 0.87 & 11.68 & 76.01 & - & - & 11.45 & - \\
\hline Ripper (default) & $S d f$ & 0.88 & 12.81 & 76.35 & - & - & 14.2 & - \\
\hline Ripper (default) & $A w f$ & 0.84 & 9.76 & 92.34 & - & - & 9.4 & - \\
\hline Ripper (default) & $S w f$ & 0.88 & 11.13 & 93.27 & - & - & 10.8 & - \\
\hline Ripper (default) & All & 0.89 & 12.56 & 311.92 & - & - & 11.6 & - \\
\hline Ripper (min. rule weight) & Exi & 0.68 & 2.10 & 4.98 & - & - & 2.52 & - \\
\hline Ripper (min. rule weight) & $A d f$ & 0.72 & 3.32 & 25.26 & - & - & 3.15 & - \\
\hline Ripper (min. rule weight) & $S d f$ & 0.70 & 3.71 & 22.21 & - & - & 2.75 & - \\
\hline Ripper (min. rule weight) & $A w f$ & 0.72 & 3.30 & 31.19 & - & - & 2.85 & - \\
\hline Ripper (min. rule weight) & $S w f$ & 0.74 & 3.87 & 32.02 & - & - & 3.1 & - \\
\hline Ripper (min. rule weight) & All & 0.75 & 3.72 & 107.92 & - & - & 3.1 & - \\
\hline SECPI & Exi & $\underline{0.93}$ & $\underline{1.66}$ & 3.35 & - & - & - & 0.75 \\
\hline SECPI & $A d f$ & 0.97 & 3.20 & 2.27 & - & - & - & 0.94 \\
\hline SECPI & $S d f$ & $\underline{0.96}$ & $\underline{2.18}$ & 1.88 & - & - & - & 0.9 \\
\hline SECPI & $A w f$ & 0.95 & 3.50 & 3.57 & - & - & - & 0.88 \\
\hline SECPI & $S w f$ & 0.96 & 3.59 & 2.93 & - & - & - & 0.92 \\
\hline SECPI & All & 0.97 & 8.46 & 8.55 & - & - & - & 0.96 \\
\hline
\end{tabular}


Table 10 Results of the experimental evaluation comparing SECPI with C4.5 and RIPPER averaged over clustering techniques and datasets, replicated with a cluster number of 8

\begin{tabular}{|c|c|c|c|c|c|c|c|c|}
\hline 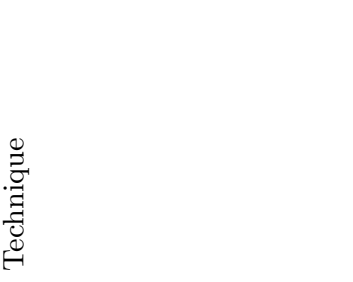 & 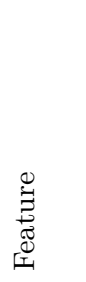 & 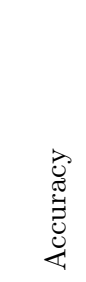 & 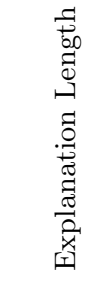 & 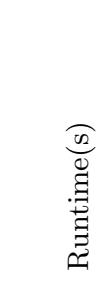 & 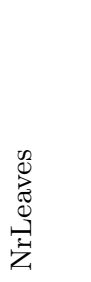 & 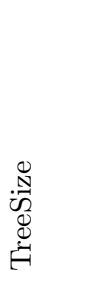 & 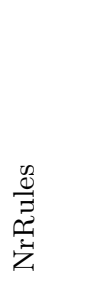 & 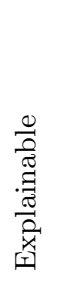 \\
\hline C4.5 (default) & Exi & 0.80 & 4.80 & 3.04 & 12.69 & 24.39 & - & - \\
\hline C4.5 (default) & $A d f$ & 0.91 & 6.92 & 19.90 & 25.17 & 49.33 & - & - \\
\hline C4.5 (default) & $S d f$ & 0.91 & 7.68 & 20.91 & 26.07 & 51.14 & - & - \\
\hline C4.5 (default) & $A w f$ & 0.90 & 6.35 & 21.98 & 19.19 & 37.39 & - & - \\
\hline C4.5 (default) & $S w f$ & 0.92 & 6.69 & 22.16 & 22.72 & 44.44 & - & - \\
\hline C4.5 (default) & All & 0.93 & 6.99 & 72.83 & 23.28 & 45.56 & - & - \\
\hline C4.5 (min. leaf size) & Exi & 0.76 & 2.99 & 1.53 & 4.15 & 7.31 & - & - \\
\hline C4.5 (min. leaf size) & $A d f$ & 0.83 & 3.49 & 8.72 & 5.28 & 9.56 & - & - \\
\hline C4.5 (min. leaf size) & $S d f$ & 0.81 & 3.51 & 7.33 & 4.97 & 8.94 & - & - \\
\hline C4.5 (min. leaf size) & $A w f$ & 0.82 & 3.49 & 10.39 & 5.06 & 9.11 & - & - \\
\hline C4.5 (min. leaf size) & Swf & 0.84 & 3.46 & 10.81 & 5.11 & 9.22 & - & - \\
\hline C4.5 (min. leaf size) & All & 0.86 & 3.59 & 33.03 & 5.33 & 9.67 & - & - \\
\hline Ripper (default) & Exi & 0.77 & 7.13 & 9.51 & - & - & 7.6 & - \\
\hline Ripper (default) & $A d f$ & 0.90 & 16.44 & 60.20 & - & - & 14.94 & - \\
\hline Ripper (default) & $S d f$ & 0.90 & 15.96 & 55.80 & - & - & 16 & - \\
\hline Ripper (default) & $A w f$ & 0.88 & 13.32 & 73.38 & - & - & 12.04 & - \\
\hline Ripper (default) & $S w f$ & 0.91 & 15.08 & 75.83 & - & - & 13.64 & - \\
\hline Ripper (default) & All & 0.92 & 16.30 & 248.24 & - & - & 14.06 & - \\
\hline Ripper (min. rule weight) & Exi & 0.69 & 2.39 & 4.72 & - & - & 2.28 & - \\
\hline Ripper (min. rule weight) & $A d f$ & 0.75 & 3.43 & 23.66 & - & - & 2.94 & - \\
\hline Ripper (min. rule weight) & $S d f$ & 0.74 & 3.83 & 20.94 & - & - & 2.74 & - \\
\hline Ripper (min. rule weight) & $A w f$ & 0.75 & 3.41 & 31.24 & - & - & 2.79 & - \\
\hline Ripper (min. rule weight) & $S w f$ & 0.76 & 4.16 & 32.74 & - & - & 2.94 & - \\
\hline Ripper (min. rule weight) & All & 0.76 & 3.78 & 106.32 & - & - & 3 & - \\
\hline SECPI & $E x i$ & $\underline{0.95}$ & $\underline{1.38}$ & 7.20 & - & - & - & 0.78 \\
\hline SECPI & $A d f$ & 0.98 & 2.43 & 10.40 & - & - & - & 0.96 \\
\hline SECPI & $S d f$ & $\underline{0.98}$ & $\underline{2.15}$ & 7.02 & - & - & - & 0.94 \\
\hline SECPI & $A w f$ & 0.97 & 2.84 & 21.69 & - & - & - & 0.91 \\
\hline SECPI & $S w f$ & 0.98 & 3.01 & 19.99 & - & - & - & 0.94 \\
\hline SECPI & All & 0.98 & 7.14 & 58.86 & - & - & - & 0.96 \\
\hline
\end{tabular}



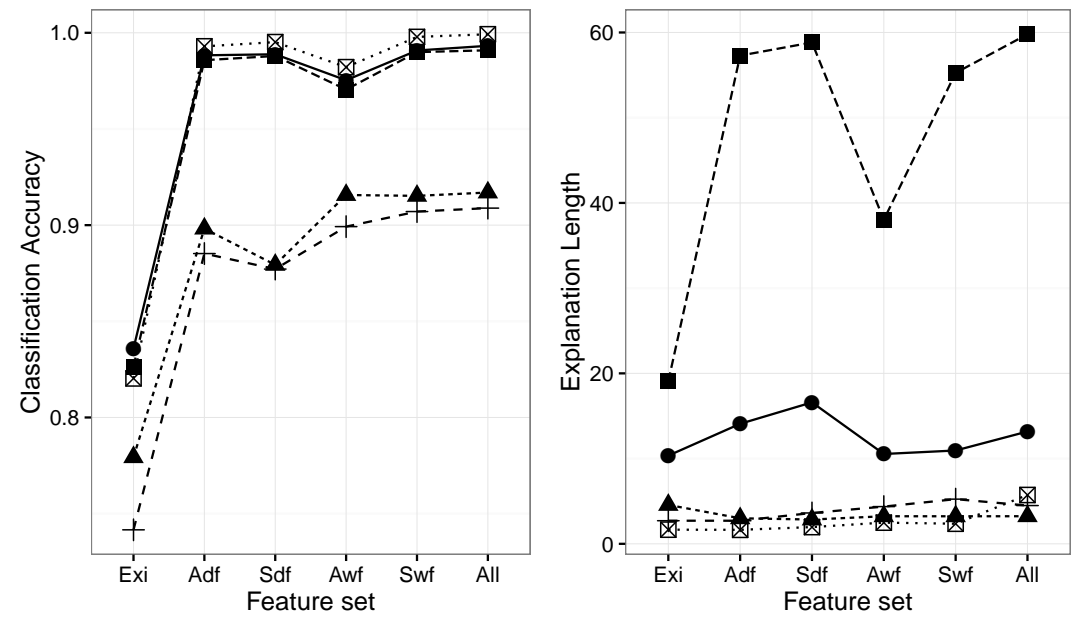

Technique - C4.5 (default) $\cdot \mathbf{A} \cdot \mathrm{C} 4.5$ (min. leaf size

- Ripper (default)-+- Ripper (min. rule size). $\cdot$ - SECPI

Fig. 7 Average accuracy (higher is better) and explanation length results (lower is better) for telecom log
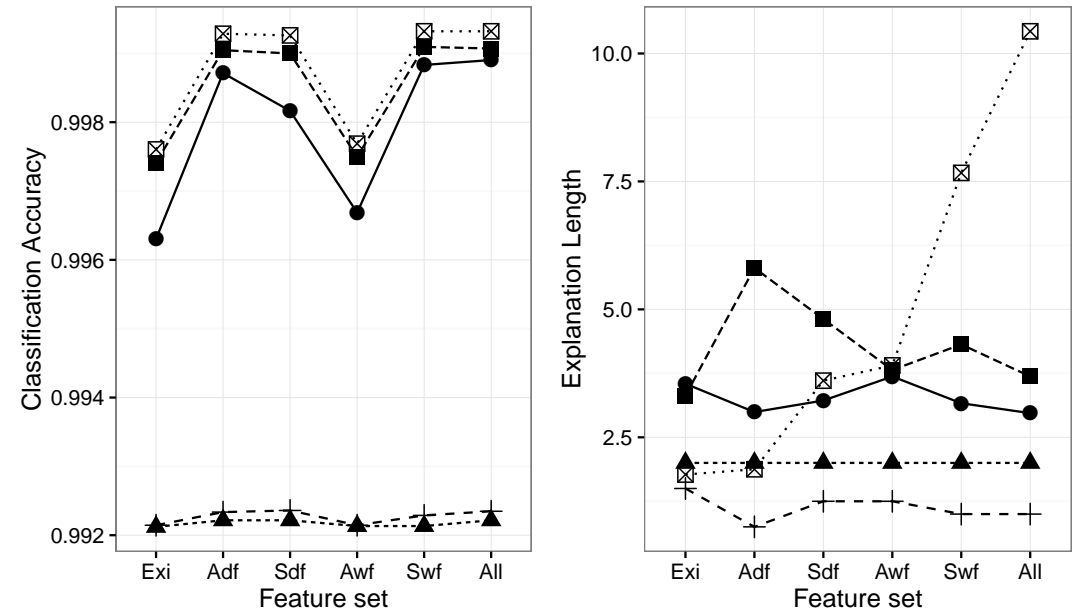

Technique $-\mathrm{C} 4.5$ (default) - - C4.5 (min. leaf size

- Ripper (default) + - Ripper (min. rule size) $\otimes \cdot$ SECPI

Fig. 8 Average accuracy (higher is better) and explanation length results (lower is better) for purchase log
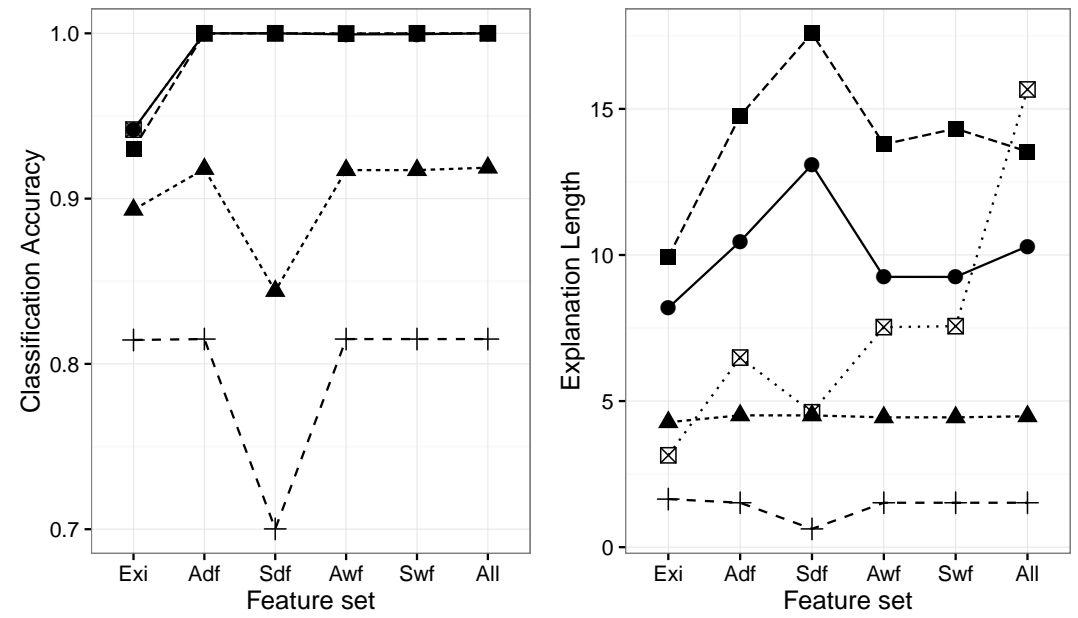

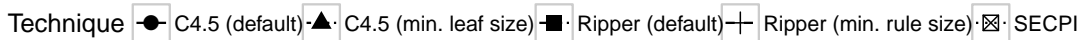

Fig. 9 Average accuracy (higher is better) and explanation length results (lower is better) for admin $\log$ 

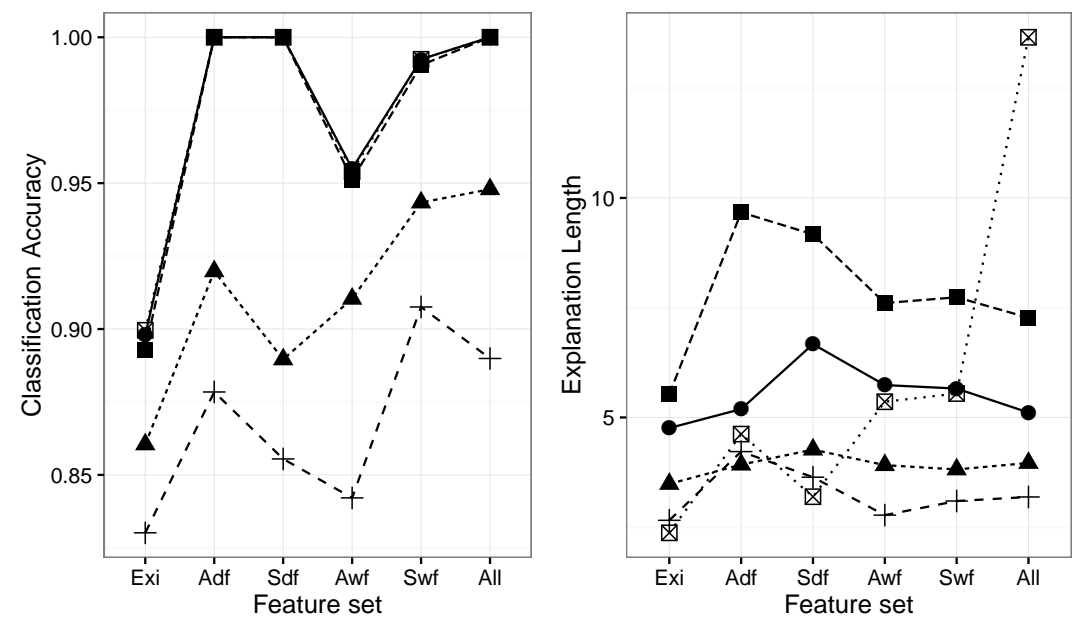

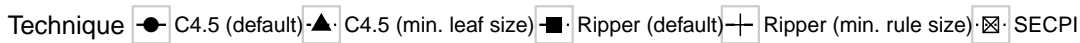

Fig. 10 Average accuracy (higher is better) and explanation length results (lower is better) for tender log
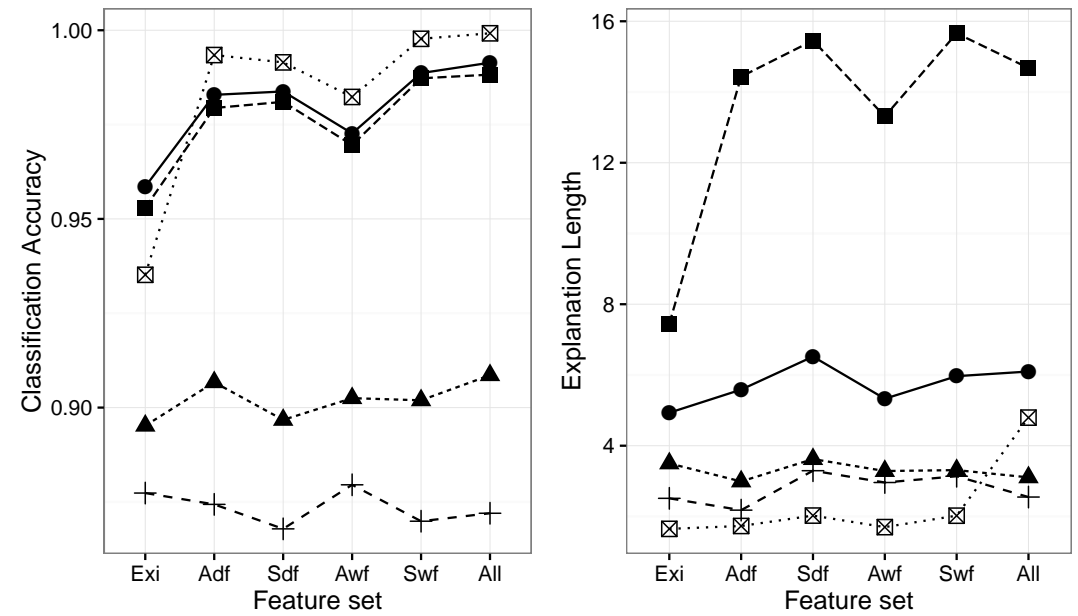

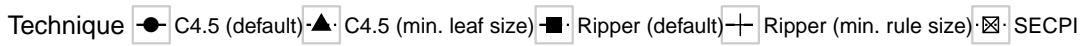

Fig. 11 Average accuracy (higher is better) and explanation length results (lower is better) for incident $\log$
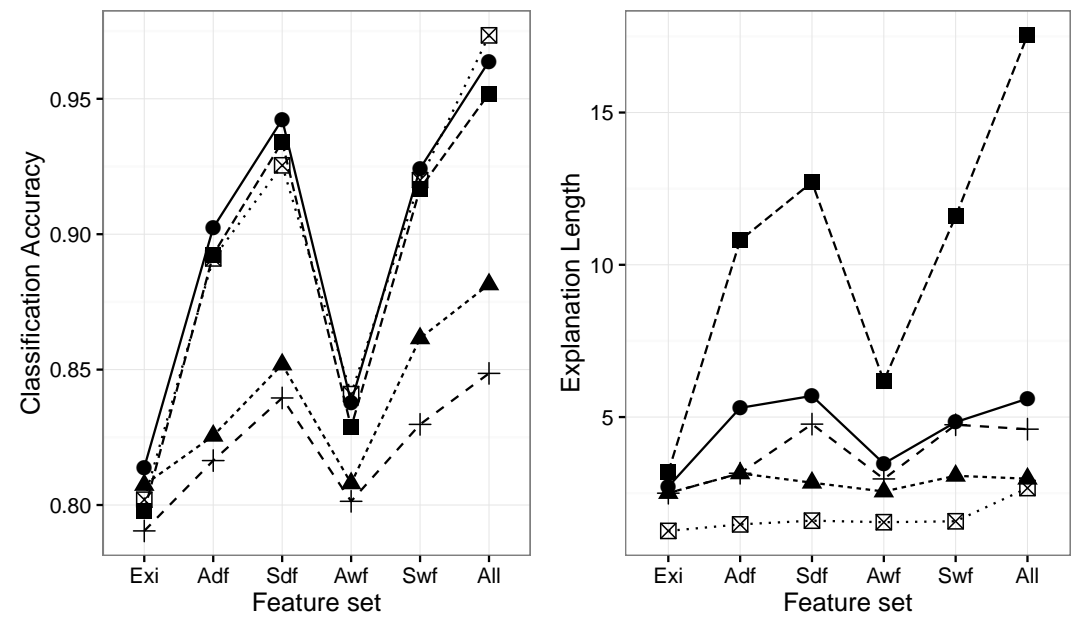

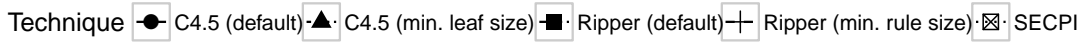

Fig. 12 Average accuracy (higher is better) and explanation length results (lower is better) for cProblem Volvo log 

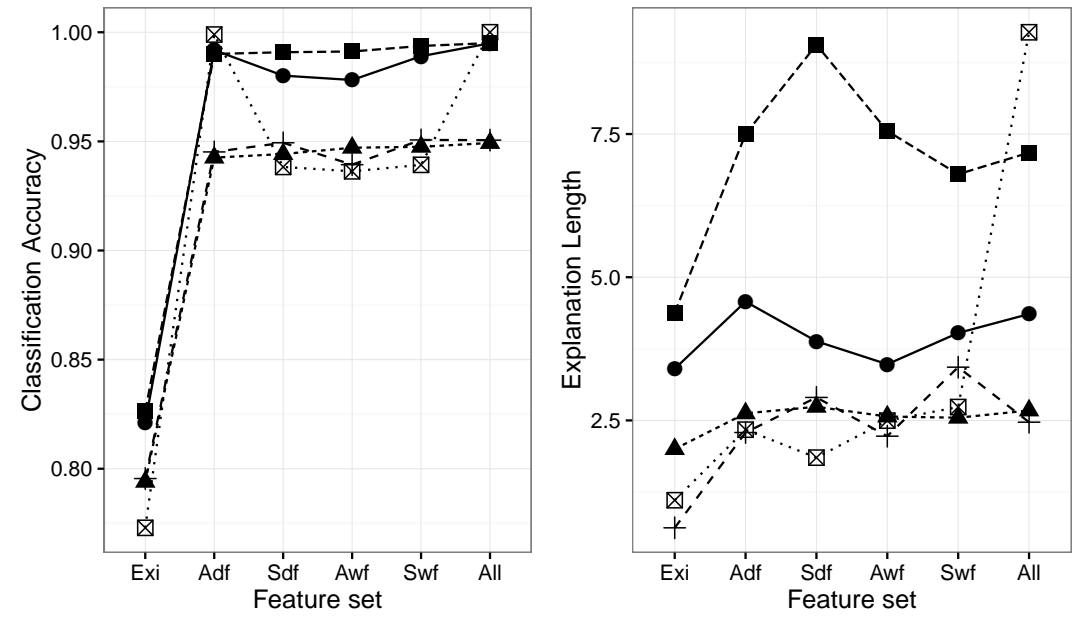

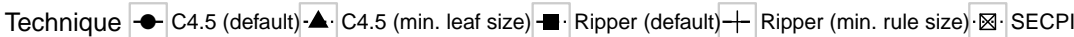

Fig. 13 Average accuracy (higher is better) and explanation length results (lower is better) for environment $\log$
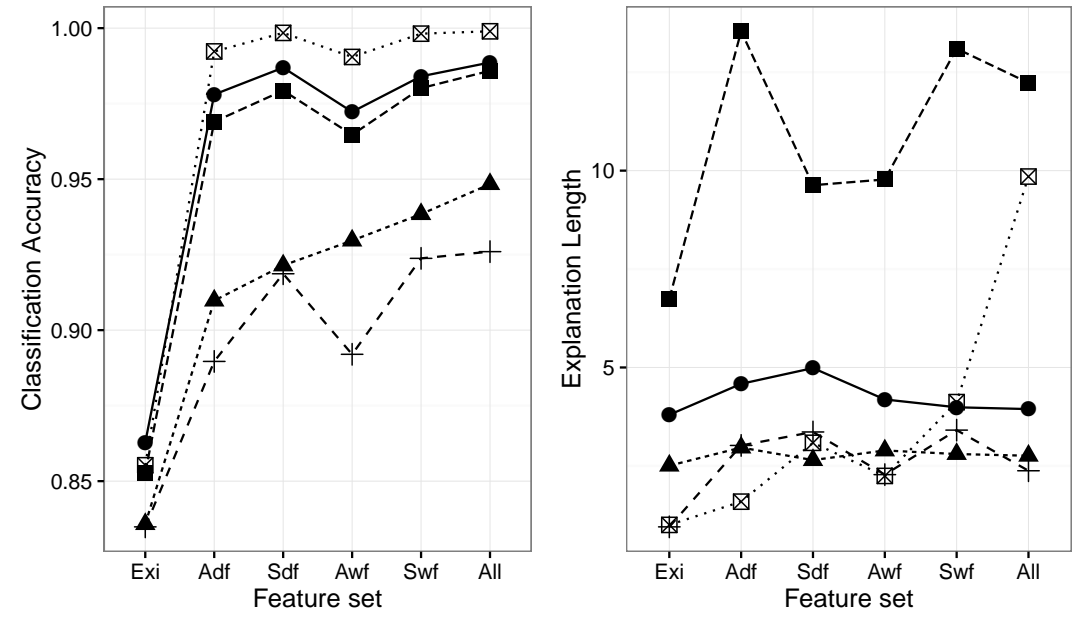

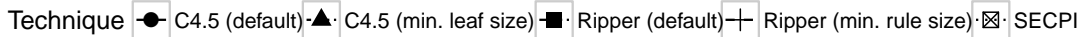

Fig. 14 Average accuracy (higher is better) and explanation length results (lower is better) for incman $\log$
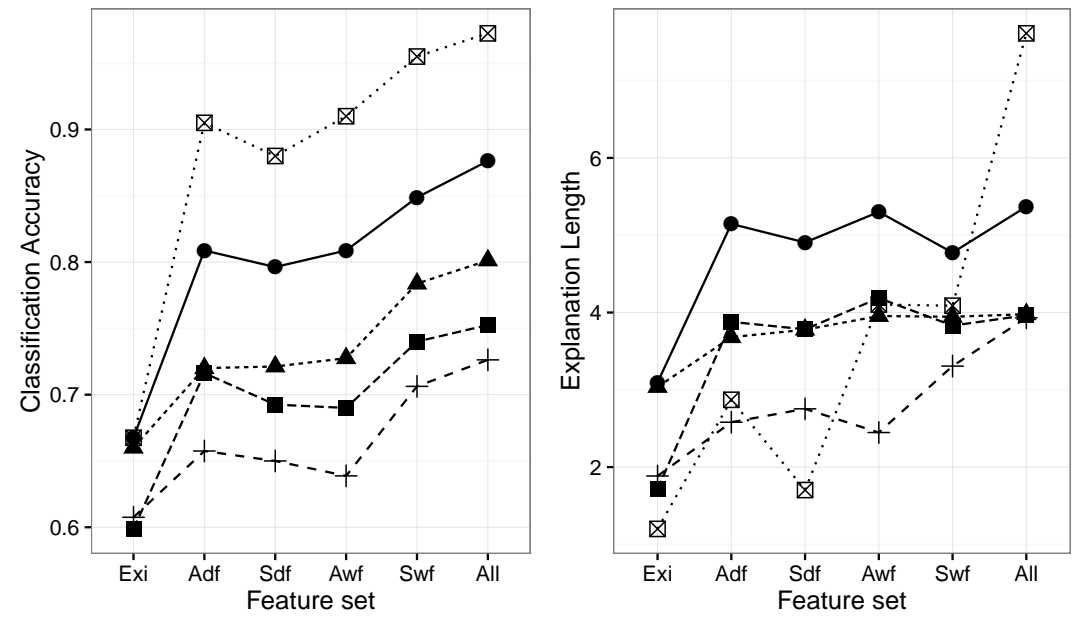

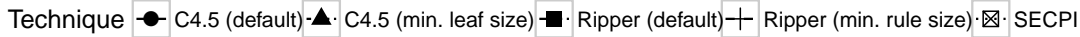

Fig. 15 Average accuracy (higher is better) and explanation length results (lower is better) for reviewing log 